\title{
Interactive Trial and Error Learning Method for Distributed Channel Bonding: Model, Prototype Implementation and Evaluation
}

\author{
Zaheer Khan and Janne J. Lehtomäki
}

\begin{abstract}
Channel bonding (CB) has been proposed as a technique to accommodate growing data rate demands in shared spectrum (SS) bands, such as in the $5 \mathrm{GHz}$ unlicensed band. Using CB a wireless user can combine multiple non-overlapping channels into one wide channel. In practice, efficient CB utilization in SS bands can be challenging as diverse owners of networks can deploy and operate heterogeneous technologies in an uncoordinated manner. This paper leverages a game theoretic learning rule for efficient distributed channel/bonding selection. Using the proposed method, each user occasionally measures channels to try out new channel/bonding selections, rejecting those selections that are erroneous in the sense that they do not lead to higher utility. Using both analytical and simulation results, we perform a Nash equilibrium assessment of the proposed method. Using various performance metrics, we analyze and compare the performance of the proposed method with a centralized solution and also with conventional distributed CB selection solutions. To realize a complete over-the-air (OTA) channel/bonding selection solution, we also implement a prototype of the proposed method on the Wireless Open-Access Research Platform (WARP). Performance of the implemented prototype is evaluated by experiments which use real OTA wireless communications.
\end{abstract}

Index Terms-Distributed channel bonding, strategic learning, game theory, heterogeneous networks, wireless research platforms, prototyping algorithms, WARP nodes.

\section{INTRODUCTION}

Wireless technologies which operate in unlicensed, and various other shared spectrum (SS) bands (where cognitive radios (CRs) enable spectrum sharing) can use channel bonding (CB) to meet consumer demand for ever-increasing wireless data rates [1]-[3].

In practice, access point (AP) deployments in SS bands are in general distributed and uncoordinated. For example, a user can individually choose to deploy an AP at his/her home or at some other place. This means that the APs make CB decisions autonomously, and the use of wider channels may increase the channel access competition for multiple APs operating in the same area. Currently, the WiFi standards consider two main methods to support $\mathrm{CB}$ [1]: Static bonding channel access (SBCA), which uses a fixed number of bonded channels and requires finding all bonded channels empty before starting a packet transmission; and dynamic bonding channel access (DBCA) in which also a user utilizes a fixed number of contiguous channels. However, different from SBCA, bonded

Z. Khan and J. J. Lehtomäki are with the University of Oulu, Finland. This work is funded by the Infotech Oulu through a grant under the framework of digital solutions in sensing and interactions and from Academy of Finland 6Genesis Flagship (grant 318927). channels are divided into a primary channel and secondary channels. When the primary channel is found free then those secondary channels that are contiguous to the primary channel and also found free at the same time are bonded together to increase the channel width.

Well known CB techniques like SBCA and DBCA do not utilize learning-based adaptation in their channel/bonding size selections. In [4], measurement-based analysis of users using $\mathrm{CB}$ has shown that while increasing the number of bonded channels increases bandwidth, it can come at the cost of greater susceptibility to interference due to the unpredictability of the interference environment in unlicensed channels. To address the limitations in existing methods, we focus on the design of a distributed learning based method using which multiple users with heterogeneous CB capabilities perform channel/bonding selections. The proposed method is different from other bonding selection methods as it utilizes adaptations in channel/bonding size selections that take into account the channel quality metric based on SINR. The proposed method enables users to select those bonding sizes that decrease the likelihood of harmful interference and increase the likelihood of achieving higher data rates. In order to realize a complete CB solution, the proposed method is not only evaluated analytically and with simulations but is also prototyped by implementing it on Wireless Open Access Research Platform (WARP) nodes [5].

A game model is usually appropriate in network scenarios where one can reasonably expect the decisions of each user to impact the outcomes relevant to the other users. We formulate the distributed channel/bonding size selection problem as a game since a user's decision to select a channel or a bonded channel impacts the channel/bonding selection outcomes for the other users. Moreover, game theory is a field of applied mathematics that describes and analyzes interactive decision situations. Game theory is useful tool for the considered problem as distributed channel/CB size selection involves interaction among uncoordinated self-motivated users. Moreover, we use interactive trial and error (ITE)-based learning framework of [6] as it provides several advantages . For example, in distributed wireless networks typically users do not have knowledge of how many other competing users are present, what are their CB capabilities, what are their payoffs, and what actions are selected by other users. In situations like these, the ITE-based learning rule offers advantage as it is completely uncoupled which means that it does not require any information about the opponents' actions or payoffs. Moreover, ITE- 


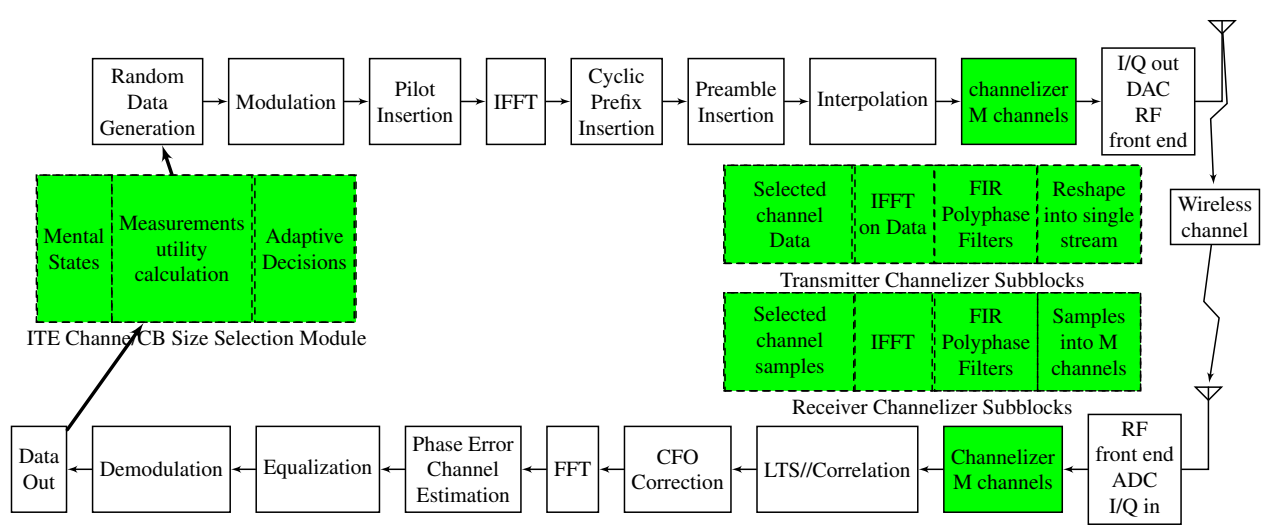

Fig. 1: Block diagram showing various components of our prototyped wireless communication system on WARP nodes using the proposed channel/bonding size selections.

based method also offers advantage as it uses a simple learning rule which unlike many other learning-based methods, such as multiarmed bandit methods, and reinforcement learning based methods, does not require to keep track of estimates of how much reward a player received from playing each action (in the form of a table or a vector list). Also, with a simple learning rule it addresses the key challenge of opponent-induced nonstationarity in wireless environment i.e. non-stationarity in the environment which is caused by changes in the action selection of the participating users. Another important advantage of ITEbased learning is that for a finite game $G$ with generic payoffs and at least one pure NE when all users use ITE-based learning with experimentation rate $\varepsilon>0$, then for all sufficiently small $\varepsilon$ a NE is played at least $1-\varepsilon$ of the time.

Our main contributions in this paper are:

- We propose an ITE-based learning method for distributed channel/bonding selections. Each user makes decisions using the proposed method in which she occasionally tries out new channel/CB size, rejecting choices that are erroneous in the sense that they do not lead to higher utility.

- Our proposed method allows users with limited information of their wireless environment to distributively arrive at those channel/bonding size selections that can satisfy their CB or rate demands. Our method does not require a user to have any knowledge of how many other users are present and what are their CB capabilities, it also does not require a user to have any information regarding what actions are selected by other users.

- Using both analytical and simulation results we show the equilibrium and convergence properties of the proposed method. We show that the CB size selection game is a generalized ordinal potential game under certain scenarios. Such games guarantee the existence of at least one pure NE. To evaluate the quality of achieved NE outcomes under various scenarios, we also present the results relating to the price of anarchy (POA), defined as the ratio of value of worst $\mathrm{NE}$ achieved by the proposed method to the value achieved by a centralized optimal solution, and the price of stability (POS), defined as the ratio of value of best $\mathrm{NE}$ achieved by the proposed method to the value achieved by a centralized optimal solution. Moreover, we also evaluate the performance of the proposed method under the following metrics: (1) number of unsatisfied users, defined as the number of users which cannot achieve their target CB size; (2) blocking rate, defined as the ratio of users who are unable to use even a single channel successfully to the total number of users; and (3) sum data rate of all users.

- We have also prototyped the proposed channel/bonding selection method on the Wireless Open-Access Research Platform (WARP) [5]. We have tested the implemented prototype in an over-the-air (OTA) environment with real wireless communications. In Fig. 1, we present a block diagram showing various components of the prototyped system. We have modified the existing WARPLab framework [7] by implementing adaptive channelizer modules which convert the single channel OFDM transmit/receive capabilities of a WARP node to a variable width multichannel OFDM transmit/receive capabilities that enable $\mathrm{CB}$. We have also implemented in the WARPLab the ITE-based CB selection module. The blocks of new implemented modules in existing WARPLab framework are drawn with green color in Fig. 1.

\section{RELATED WORK}

There are more than 20 non-overlapping unlicensed channels available in the $5 \mathrm{GHz}$ band. Moreover, spectrum sharing in new spectrum bands, such as TV white space and radar bands, will also make additional spectrum available. This availability of more spectrum has allowed the feasibility of using multiple bonded channels in various SS bands [8]-[12]

Much of the existing literature focusing on intelligent channel/CB selection methods concentrates on designs where selections are performed by a centralized entity which acts as a coordinator for the users in the network [13]. For example, different from our work, in [13], authors have considered CB assignments in shared access systems where a centralized entity can perform optimization of guard band aware CB decisions. In [8], an analytical framework was proposed to investigate the interactions between multiple overlapping local area networks that use conventional distributed CB methods, 
such as the DBCA method. Although CB in unlicensed and shared spectrum is currently actively researched, however, to the best of our knowledge, only a recent work in [14] has considered $\mathrm{CB}$ with dynamic link adaptation using the learning framework based on multi-armed bandit problem. The bandit formulation used to model decision-making in [14] is based on stationary environment perceived by a user. However, in our work, we consider more realistic opponent-induced nonstationarity in the environment, i.e. non-stationarity in the environment which is caused by changes in the action selection of the participating users [15].

We consider fully decentralised network scenarios where there is no assumption of synchronicity in decision making (or interactions) among the users and one or more users can act simultaneously. Also, we do not also assume that a user can observe other users actions or users exchange action information with one another. This means that best response dynamics [16] or asynchronous best response dynamics based solutions, such as given in [16], [17], cannot be applied to our work.

In [18], we proposed distributed and coordinated channel/bonding selection methods under SINR and collisionchannel models. However, our work in [18] ignores uncertainty in bonding size demands of users. Different from [18], to overcome the uncertainty, the proposed method has the following key ingredient: if someone experiences a payoff decrease due to external factors such as a bonding size strategy change by some other user or due to an internal factor such as a change in a user's own bonding size demand, the user starts a search for a new action, eventually settling on one with a probability that increases monotonically with her realized payoff. The distributed method in [18] also ignores strategic interactions among users. Also, different from [18], using analytical and simulation results we provide extensive NE analysis of the proposed method. Moreover, unlike [18], in this paper, a complete channel/bonding size selection solution is realized by presenting an implementation of the prototype of the proposed method. The implemented prototype is evaluated by experiments which use real OTA wireless communications.

\section{System ModeL}

\section{A. Network and CB Model}

We consider a network of $N$ distributed users (transmitter/receiver wireless links) which compete in a set $\mathcal{M}=$ $\{1,2, \cdots, M\}$ of unlicensed channels. In our model, users operate in the network under heterogeneous CB scenarios. For example, heterogeneity in terms of $\mathrm{CB}$ can be due to different CB capabilities of users, as different users may use different wireless standards. Some wireless standards may not allow $\mathrm{CB}$, and the others may vary in terms of number of channels that are allowed to be bonded [1]. We particularly focus on CB-based spectrum access techniques for scenarios where users operate over wide swathes of spectrum. In our model, $C_{i}$ represents the $\mathrm{CB}$ capability of a user $i$, where $C_{i} \in\left\{1,2, \cdots, C_{m}\right\}$ and $C_{m} \leq\left\lfloor\frac{M}{2}\right\rfloor$ is the maximum size of $\mathrm{CB}$ allowed for a user in the network. This limit is to protect the network users from too high interference. Note that $C_{i}=1$ means that the user $i$ has no bonding capability. The bonding channel size $S_{i}$ represents the number of channels utilized by $i$ and $S_{i} \leq C_{i}$.

When a user with $C_{i}>1$ finds two or more (contiguous) channels suitable for communications, she may bond these channels into a single channel and transmit a larger packet. When a user with $C_{i}>1$ utilizes $S_{i}=1$ it means that the user $i$ is using a single channel, $S_{i}=2$ means two single channels are bonded into one, and so on.

We also consider non-contiguous CB scenarios. The noncontiguous bonding increases the number of ways a user can bond channels as it allows a user to take advantage of bonding across multiple separate channel sets, such as in $5 \mathrm{GHz}$ spectrum where two unlicensed channel sets are separated by radar channels. This means that the non-contiguous $\mathrm{CB}$ can outperform contiguous $\mathrm{CB}$ for the scenarios where channels available for bonding are scattered across multiple channels. Let us consider that the total number $M$ of the unlicensed channels that can be used are allocated in $\kappa$ chunks, where $\kappa=1$ chunk means that all are allocated contiguously together and the unlicensed frequency channels that can be used are not fragmented, $\kappa=2$ chunks mean that the $M$ frequency channels are not allocated contiguously but there is a gap between the two different sub-bands, $\kappa=3$ chunks mean that there are two gaps between a total of three different subbands, and so on. For tractability, let's assume that when the channels are allocated in $\kappa>1$ chunks, there are $\left\lfloor\frac{M}{\kappa}\right\rfloor$ channels allocated in each of the first $\kappa-1$ chunks and the remaining $M-\left\lfloor\frac{M}{\kappa}\right\rfloor(\kappa-1)$ channels are allocated in the last $\kappa$ th chunk.

The number of available channel/bonding selections to choose from for a user $i$ with contiguous $\mathrm{CB}$ capability $C_{i}$ and $M$ channels in $\kappa$ chunks is

$$
\begin{aligned}
& M_{a}^{c}=\sum_{S_{i}=1}^{\min \left(\left[C_{i},\left\lfloor\frac{M}{\kappa}\right]\right]\right)}\left(M-\left(S_{i}-1\right) \kappa\right)+ \\
& \sum_{S_{i}=\min \left(\left[C_{i},\left\lfloor\frac{M}{\kappa}\right\rfloor\right]\right)+1}^{\min \left(\left[C_{i},\left(M-\left\lfloor\frac{M}{\kappa}\right\rfloor(\kappa-1)\right]\right)\right.}\left(M-\left\lfloor\frac{M}{\kappa}\right\rfloor(\kappa-1)-\left(S_{i}-1\right)\right)
\end{aligned}
$$

The number of available channel/bonding selections to choose from for a user $i$ with non-contiguous $\mathrm{CB}$ capability $C_{i}$ is simply

$$
M_{a}^{n c}=\sum_{S_{i}=1}^{C_{i}}\left(\begin{array}{c}
M \\
S_{i}
\end{array}\right)
$$

Using Eq. 1 and Eq. 2, we can find the increase in the number of ways a user can select channels when it has non-contiguous CB capability $C_{i}$ by

$$
\Delta_{M}=M_{a}^{n c}-M_{a}^{c}
$$

It can be seen that $\Delta_{M}$ increases with increase in $\kappa$. This gain does not come without additional costs. For example, the non-contiguous $\mathrm{CB}$ increases the likelihood of adjacent channel interference (ACI) for a user. We provide an upper bound on the number of ways in which a user can experience ACI on her selected channels for the non-contiguous CB case. Let $\alpha_{i}$ represent the number of ways in which a user experiences ACI on her channels then for the non-contiguous 
case $\alpha_{i} \leq\left(v_{i}+1\right) 2$, where $v_{i}$ represents the number of gaps between channels selected by the user for bonding. It is easy to see that for the contiguous CB it is simply $\alpha_{i} \leq 2$. Moreover, the non-contiguous $\mathrm{CB}$ can also pose other challenges on the radio front-end and requires increased computational resources. For example, tight synchronization is needed when non-contiguous bonding is used. This is due to the reason that non-contiguous bonding requires computationally expensive operations called signal decomposition at the transmitter side and then recomposition at the receiver side. If the baseband is mapped to a contiguous physical band, there is no need for the decomposition and recomposition operations.

We denote the set of all possible selections for a user $i$ with given $C_{i}$ and $M$ channels by $\Sigma_{i}$. For example, for the contiguous bonding case and for $\kappa=1, \Sigma_{i}$ is given as:

$$
\begin{aligned}
& \Sigma_{i}=\{\overbrace{\{\{1\},\{2\},\{3\}, \ldots,\{M\}\}}^{\text {Set of } S_{i}=1 \text { selections }}, \overbrace{\{1,2\},\{2,3\},\{3,4\}, \ldots . .\}}^{\text {Set of } S_{i}=2 \text { selections }} \\
& \overbrace{\left\{1,2, \ldots, C_{i}\right\},\left\{2,3, \ldots, C_{i}+1\right\}, \ldots \ldots .}^{\text {Set of } S_{i}=C_{i} \text { selections }}\}
\end{aligned}
$$

Users communicating with fixed transmission powers is the typical mode of operation for uncoordinated wireless networks, such as networks deployed in unlicensed spectrum [1]. In our model, we consider that each user can use fixed total transmit power $P$. This means that when a user selects a single channel then she uses her total power $P$ for a transmission in that single channel, and when she selects multiple single channels and bonds them into one then she uses the same power $P$ to transmit in the bonded channel.

\section{B. SINR-based Access Model with ACI}

One of the most commonly studied access models for wireless networks is the SINR-based access model [19]. Consider a wireless channel where the received signal strength at a receiver $i$ from transmitter $j$ is [20]:

$$
P_{r, i j}=P_{0, i j}\left(\frac{d_{i j}}{d_{0, i j}}\right)^{-\alpha},
$$

where $d_{i j} \geq d_{0, i j}$ is the distance of receiver $i$ from transmitter $j$. The reference received power level $P_{0, i j}$ at the close in distance $d_{0, i j}=\max \left\{\frac{2 D_{i}^{2}}{\lambda_{i}}, D_{i}, \lambda_{i}\right\}$ of receiver $i$ from transmitter $j$ is [20]:

$$
P_{0, i j}=\frac{P_{t, j} G_{t, j} G_{r, i} \lambda_{i}^{2}}{\left(4 \pi d_{0, i j}\right)^{2}},
$$

where $D_{i}$ is the receiver antenna length, $\lambda_{i}$ is the wavelength of the center frequency of the channel, $P_{t, j}$ and $G_{t, j}$ are the transmit power and transmitter antenna gains, respectively, of transmitter $j$, and $G_{r, i}$ is the receiver antenna gain. Under the SINR-based access model, when a user $i$ with $C_{i}>1$ finds two or more channels suitable for communications, she bonds these channels into a single channel and transmits a larger packet. The SINR at the receiver of user $i$ is then calculated as follows:

$$
\gamma_{i}=\frac{P_{r, i j}}{\left(\sum_{k=1, k \neq j}^{I_{i}} P_{r, i k}\right)+N_{0} S_{i} W},
$$

where $P_{r, i k}$ is the interference power from transmitter $k$ at receiver $i$ (taking into account overlap of channel selection), $I_{i}$ represents the number of other users that have selected the same channel(s) as the considered user $i, N_{0}$ is noise power spectral density, and $W$ is the bandwidth of the single channel. The Shannon rate $R_{i}$ for a single channel of bandwidth $W$ is

$$
R_{i}=W \log _{2}\left(1+\gamma_{i}\right)
$$

Under the SINR model, a user can successfully communicate as long as the received SINR is greater than or equal to a defined threshold SINR value $\gamma_{0}$. Loss of communication only occurs when $\gamma_{i}<\gamma_{0}$. The value of $\gamma_{0}$ can vary from one wireless system to another. It depends on various parameters such as the transmit power, bandwidth utilized, etc. In practice, $\gamma_{0}$ should be selected to achieve reasonable communication performance for a user. In our model we also take into account the impact of ACI. For example, consider the situation at a receiver that is affected by one interferer. Suppose that the interferer is transmitting on channels 1 and 2 and the receiver is receiving on 2,3, and 4 . Assume that the interferer divides her transmit power equally over channels 1 and 2, the receiver can directly get interference impact from $50 \%$ of the interferer's transmit power. The receiver may also get ACI from interferer's channel 1, corresponding to $50 \%$ of the interferer's transmit power scaled down by the ACI factor (ACI factor will be 0 when it is not modeled). For example, if the ACI factor is $0.05(-13 \mathrm{~dB})$, the receiver for the above mentioned scenario can get interference impact from $50 \%+50 \% * 0.05=52.5 \%$ of the interferer's power. If the receiver is tuned to channel 3 only, it would only receive ACI from channel 2 corresponding to $50 \% * 0.05=2.5 \%$ of the interferer's power. If ACI is not modeled, receiver on channel 3 only would not get any power from the interferer on channels 1 and 2 .

\section{PRoposed LEARNING B Y ITE FOR Channel/BONDING SizE SELECTIONS}

\section{A. Game Formulation}

We formulate the distributed channel/bonding size selection problem as a demand-based ITE learning game $\mathcal{G}=$ $(\mathcal{N}, \mathcal{A}, \mathcal{U}, \mathcal{D})$, where $\mathcal{N}$ is the set of users, $\mathcal{A}=A_{1} \times \cdots \times A_{N}$ with $\mathcal{A}_{i}$ the set of actions for each user $i$. An action of user $i$ is denoted by $a_{i}$, and a vector of strategy profile of all users is given by $\mathbf{a}=\left(a_{1}, a_{2}, \cdots, a_{N}\right)$. The set of actions that a user $i$ uses for selecting a channel/bonding size is given by $\sum_{i}$. In a given time-step, we also allow the users to opt out of playing by selecting the null action, i.e., not select any channel/bonding size for communication and stay quiet. When a user selects the null action we say she has selected the virtual channel which is denoted by $v=0$. In other words, the total set of available actions is $A_{i}=\{0\} \cup \sum_{i} . \mathcal{U}=u_{1} \times \cdots \times u_{N}$, with $u_{i}: \mathcal{A} \longrightarrow \mathbb{R}$ is the real valued utility function for each user $i$ that measures her degree of satisfaction as a function of the combination of all users choices, and $\mathcal{D}=D_{1} \times \cdots \times D_{N}$, where $D_{i}$ is the bonding size requirement (demand) of each user $i$. Distributed users in the network can have heterogeneous 


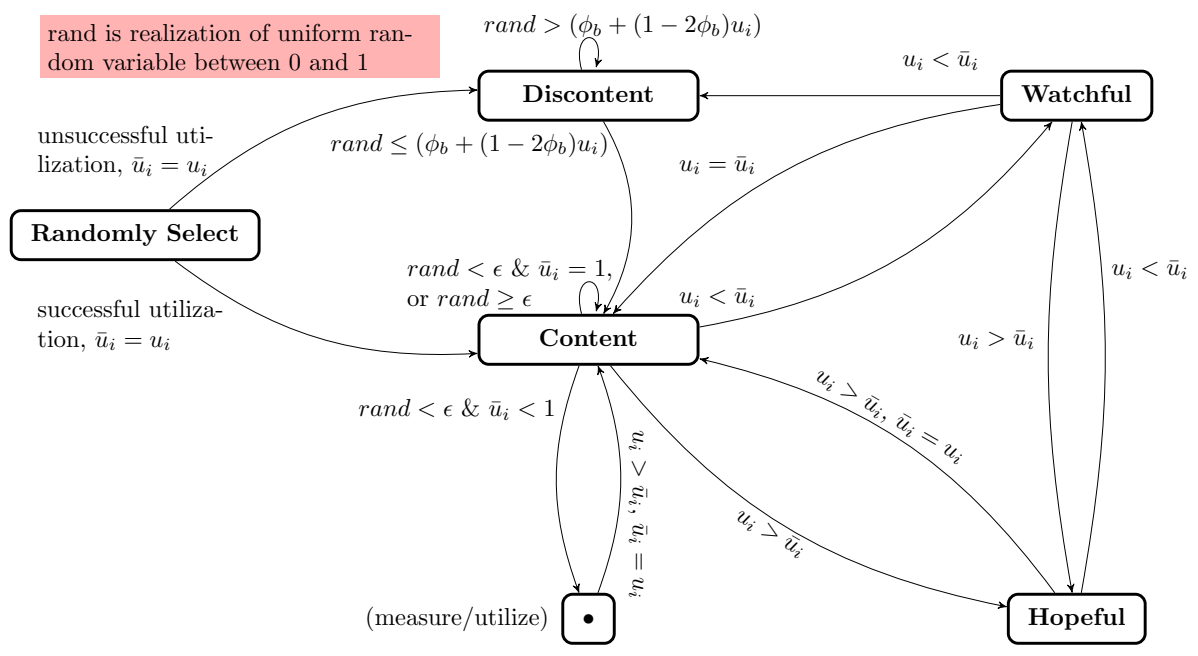

(a)

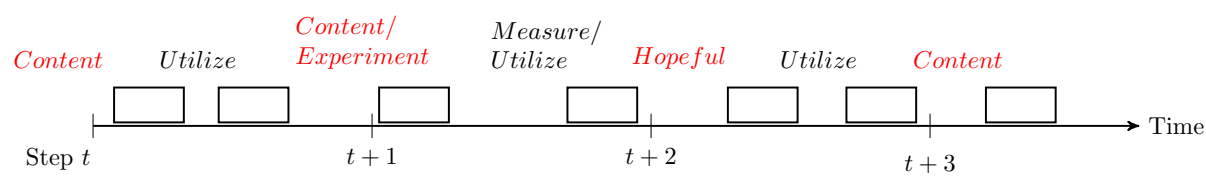

(b)

Fig. 2: a) The structure of ITE mood state transitions for a given user $i$ with benchmark payoffs, and current period payoffs. b) An example of possible state transitions for a user in content state at step $t$.

CB demands due to their different CB capabilities, and/or due to different rate requirements of applications used by the users.

\section{B. CB-demand based utility}

A significant portion of game-theoretic literature has focused on utility maximization, however, there are several works, such as [21], [22] that have shown that utility maximization does not always seem plausible as a description of how players actually make decisions in complex environments. One alternative to utility maximization approach is a "satisfactory (demand-based) payoff" approach for a player which assumes that the player tends to repeat satisfactory actions, and explores alternatives to unsatisfactory actions [21], [22]. Influences of satisfaction-based behavior on outcomes are studied in various economic models (see [21], [22], and references therein). Recent works, such as [23], have also used utility satisficing as a relaxation of utility maximizing in the context of a multi-armed bandit problem.

The utility function of a user $i$ is given by:

$$
u_{i}= \begin{cases}\frac{S_{i}}{D_{i}}, & \gamma_{i} \geq \gamma_{0}, a_{i} \in \Sigma_{i} \\ -c, & \gamma_{i}<\gamma_{o}, a_{i} \in \Sigma_{i} \\ 0, & a_{i}=0 .\end{cases}
$$

where $S_{i} \in\left\{1,2, \cdots, D_{i}\right\}$, and $D_{i}$ is the CB demand of user $i$. The utility function is meaningful from the perspective of users performing channel/bonding selections in a wireless system as it captures usefulness a user gets from the selected channel/bonding action. When a user selects an action and is able to communicate meaningful information, i.e., signal-tointerference-plus-noise-ratio (SINR) is greater than or equal to some defined threshold value $\left(\gamma_{i} \geq \gamma_{0}\right)$, then it achieves a utility proportional to the ratio of the number of channels it has been able to bond to its bonding demand. This ratio captures users preference of bonding more channels when using them they can communicate meaningful information. When a user selects an action and using this action it is not able to communicate meaningful information, i.e., $\gamma_{i}<\gamma_{0}$, then the utility is $-c$, where $0<c \ll 1$ is the penalty of transmitting (wasting power) despite the fact that the user cannot communicate meaningful information, and when the user cannot communicate meaningful information and selects the null action (does not transmit) then the utility is zero. Moreover, as our work also focuses on practical prototype implementation of the proposed channel/CB method, the proposed utility is also very suitable for such practical implementation purposes as it does not require complex computational operations, such as computation of logarithmic function. For example, it can be implemented as one multiplication operation when a small pre-calculated look-up table (LUT) is used. This limits the complexity of processing on a real system.

\section{Dynamics of Channel/Bonding Size Selections Using ITE}

Each user $i$ has a mental state at a given time instant which is given by $z_{i}=\left(m_{i}, \bar{a}_{i}, \bar{u}_{i}, S_{i}\right)$, where $m_{i}$ is user $i$ 's mood, which may be content, hopeful, watchful, or discontent, $\bar{a}_{i}$ is $i$ 's benchmark action, $\bar{u}_{i}$ is $i$ 's benchmark utility, and $S_{i}$ is $i$ 's selected CB size. A state $z=(\mathbf{m}, \overline{\mathbf{a}}, \overline{\mathbf{u}}, \mathbf{s})$ of the process specifies the state $z_{i}$ of each user, where each of the four components is a $\mathrm{N}$-length vector describing the user moods, benchmark actions, benchmark utilities, and CB sizes. 
The ITE mood state transitions for a given user $i$ with benchmark action $\bar{a}_{i}$, benchmark utility $\bar{u}_{i}$, current action $a_{i}$, and current utility $u_{i}$ are illustrated in Fig. 2a. An example of possible state transitions for a user in content state are illustrated in Fig. 2b. We next explain the important steps involved in the proposed method using ITE in details:

- Upon becoming active, a user starts with single channel selection (i.e., $S_{i}=1$ ), and selects a channel randomly with uniform probability for utilization. One of two possibilities occur: 1) Utility is greater than zero. A user $i$ will update her benchmark action $\bar{a}_{i}$ to her current action $a_{i}$, i.e., $\bar{a}_{i}=a_{i}$, the user will update benchmark utility $\bar{u}_{i}$ to current realized utility, i.e., $\bar{u}_{i}=u_{i}$, and the user will update her mood to content; 2) Utility is less than zero. A user $i$ will update her benchmark action to $\bar{a}_{i}=a_{i}$ and benchmark utility to $\bar{u}_{i}=-c$, and the user will update her mood to discontent.

- Greater than zero utility means that with her single selected channel the user $i$ can successfully communicate and obtains initially the utility $u_{i}=\frac{1}{D_{i}}$ (see Eq. 9), otherwise, initially, she obtains the utility $u_{i}=-c$

- When content, a user $i$ will experiment in the next timestep with probability $\varepsilon$, and with probability $1-\varepsilon$ she will not experiment.

- When the user $i$ experiments in content mood, she checks whether her benchmark utility is $\bar{u}_{i}<1$, if yes, she either randomly experiments with a new channel by actually going there, or she first performs channel quality measurements and based on these measurements decides whether to actually go to the new channel(s) for experiment, otherwise for $\bar{u}_{i}=1$, she remains in the content mood and keeps utilizing benchmark action $\bar{a}_{i}$ as current action.

- Channel quality measurements: To obtain quality of other selected channel, the user measures interference plus noise level in that channel. One way to measure interference plus noise level on a channel is to use minimum value processing (MVP) technique presented and prototyped by us in [24]. The MVP technique operates in real-time directly on the energy detection outputs which are an unknown combination of other users signals (interference) and noise of the measured channel. In simple words, the MVP technique in [24] uses the fact that mostly signal samples are not randomly distributed among all the received samples but are clustered together. For example, samples corresponding to the interference represent packet transmissions of other users and are naturally clustered together in time domain. Also, this means that noise-only samples are clustered together. When we use minimum operation, we can find the energy detector output with noise-only samples, and use it to estimate noise power. When the received samples are not noise-only, i.e., they exceed some signal threshold value, MVP estimates interference power. The sum of estimated noise and interference power are used to estimate the interference-plus-noise on measured channels. The estimate of received signal strength at her receiver from own transmissions is used to measure the channels' quality in terms of SINR. An action $a_{i}$ which represents increase in bonding size by 1 is selected if it satisfies the desired channel quality, otherwise, the user will use the channel specified by her benchmark $\bar{a}_{i}$ for access, and will remain in content mood. Note that it is possible that the bonding size is increased by combining a new channel with the currently utilised channel(s) for access.

- Action update: In both random experimentation and measurement based experimentation cases, when an action is selected then in the next time-step, the current action $a_{i}$ is updated to the selection, and the utility $u_{i}$ is realized using this action. If the realized utility is greater than the benchmark utility, i.e., $u_{i}>\bar{u}_{i}$, a user $i$ will update her current benchmark action $\bar{a}_{i}=a_{i}$ and benchmark utility $\bar{u}_{i}=u_{i}$, the user will remain in content mood, otherwise, the user will stop using the current action $a_{i}$ the user will reuse $\bar{a}_{i}$ as her current action $a_{i}$, and will remain in the content mood.

When a user does not experiment in the content mood, in the next round, she utilizes the benchmark action $\bar{a}_{i}$ as her current action $a_{i}$, if her realized utility $u_{i}$ is equal to the benchmark utility $\bar{u}_{i}$ she remains in content mood. When not experimenting, the actions of other users may influence $i$ 's mood to change, by causing her realized utility $u_{i}$ to change by either increasing or decreasing. If $i$ does not experiment, and realized utility $u_{i}<\bar{u}_{i}$, then $i$ becomes watchful. On the other hand, if $i$ does not experiment, and $u_{i}>\bar{u}_{i}$, then $i$ becomes hopeful.

- When a user is hopeful, in the next round she plays her current action $a_{i}$ with probability 1 . If $u_{i}>\bar{u}_{i}$, then the user becomes content and updates her benchmark utility $\bar{u}_{i}=u_{i}$ and benchmark action $\bar{a}_{i}=a_{i}$, if $u_{i}<\bar{u}_{i}$, then the user becomes watchful, otherwise the user becomes content with her current benchmarks.

- When a user is watchful, in the next round she plays her current action $a_{i}$ with probability 1 . If $u_{i}<\bar{u}_{i}$, then the user becomes discontent, if $u_{i}>\bar{u}_{i}$ the user becomes hopeful, otherwise the user becomes content with her current benchmarks.

- When a user is discontent, she selects randomly a channel of bonding size 1 out of those channels that is/are not currently utilized by the user. She updates the utility $u_{i}$ realized by using this action. The user utilizes a response function given by

$$
\Phi_{R}=\phi_{b}+\left(1-2 \phi_{b}\right) u_{i}
$$

where $0<\phi_{b}<0.1$ and $\Phi_{R} \in(0,1)$. With probability $\Phi_{R}$ the user spontaneously becomes content. She updates current benchmark action $\bar{a}_{i}=a_{i}$ and utility $\bar{u}_{i}=u_{i}$ if she obtains $u_{i}>0$, otherwise, she updates $\bar{a}_{i}=0$ (null action) and utility $\bar{u}_{i}=0$. With probability $1-\Phi_{R}$ the user remains discontent and repeats the process. 


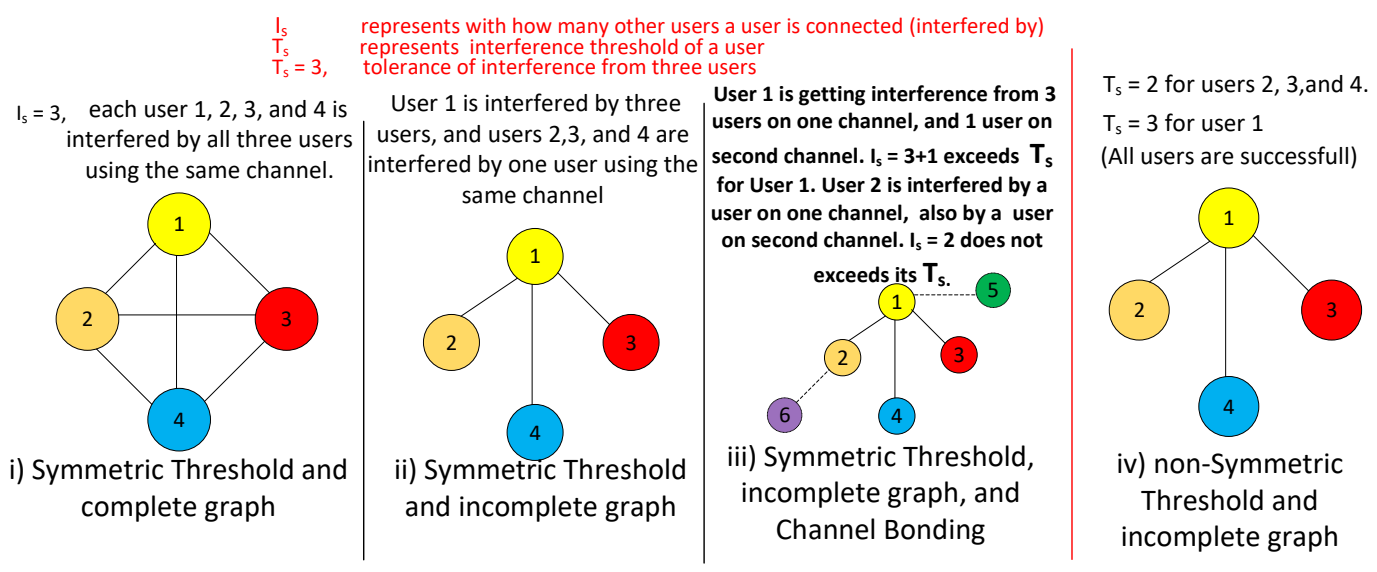

Fig. 3: Examples showing interference graph representation of network with various threshold requirement scenarios. Each circle represents a user. $I_{S}$ is used to represent: for each example scenario with how many other users a user is connected (interfered by).

\section{Equilibrium Properties}

The solution concept for the channel/bonding size selection that we focus on is Nash equilibrium.

Definition 1. A pure strategy Nash equilibrium (NE) is a profile of actions such that each action is a best response $(B R)$ to the rest of the actions. The NE outcome can be formally given as follows: An action profile $\mathbf{a}=\left(a_{1}, a_{2}, \cdots, a_{N}\right)$ of an $N$ user game is a NE if for each user $i$, we have,

$$
u_{i}\left(a_{i}, \mathbf{a}_{-i}\right) \geq u_{i}\left(\hat{a}_{i}, \mathbf{a}_{-i}\right), \forall \hat{a}_{i} \in A_{i}, \forall i \in \mathcal{N} .
$$

That is, the action $a_{i}$ of each user $i$ is her BR to the actions $\mathbf{a}_{-i}$ of all other users.

This concept has been widely applied in the study of uncoordinated wireless networks. We next analytically show that the ITE dynamics stabilizes to a NE under different simplified network scenarios. It is important to note that analytically showing whether and when the ITE dynamics stabilizes to a $\mathrm{NE}$ is challenging for the considered fully distributed network model of $N$ wireless links with heterogeneous CB capabilities, which operate at different distances from one another, which can be affected by asymmetric channel/interference conditions, which know only their own payoff, and which do not use control channel for signaling. The scale, complexity and randomness of such a distributed wireless network makes her theoretical modeling/analysis challenging. However, in Section VI, we will describe our simulation results that evaluate whether and when the ITE dynamics stabilizes to a NE under the complex and more realistic system model considered by us in Section III.

Observation IV.1. A commonly used approach to make the algorithmic analysis tractable is to approximate the underlying wireless network as an undirected interference graph, where vertices represent users, and an undirected edge connecting two users represents that these two users are within interference range of one another. In other words, an edge between two users represents that they can generate interference to each other under the SINR model.

Observation IV.2. In the undirected interference graph rep- resentation of the network, one can define the SINR threshold requirement with respect to a user to be an integer value. One can determine whether the SINR threshold requirement of a user is satisfied by counting the number of edges which represent number of neighbors that are using the same channel as herself and causing interference to her. Examples of various threshold requirements and threshold satisfaction scenarios are illustrated in Fig. 3.

Before presenting the formal proof that shows the existence of pure strategy NE, we first make an important observation and then introduce some definitions that can help understand some of the concepts used in the derivation of the proof.

Definition 2. An $N$ player game is a generalized ordinal potential game if it admits an ordinal potential $\Phi$ which satisfies the following property $\Phi: \mathcal{A} \rightarrow \mathbb{R}$ such that $\forall i \in \mathcal{N}$, all $a_{i}, a_{i} \in A_{i}$, and all $\mathbf{a}_{-i} \in A_{-i}$ :

$$
u_{i}\left(\hat{a}_{i}, \mathbf{a}_{-i}\right)-u_{i}\left(a_{i}, \mathbf{a}_{-i}\right)>0 \Longrightarrow \Phi\left(\hat{a}_{i}, \mathbf{a}_{-i}\right)-\Phi\left(a_{i}, \mathbf{a}_{-i}\right)>0 .
$$

In other words, an increase or decrease in a user's utility due to the user's unilateral deviation implies an increase or decrease in the potential function.

Proposition IV.1. Under the undirected interference graph representation, the channel/CB selection game $\mathcal{G}$ is a generalized ordinal potential game.

Proof: The generalized ordinal potential function for the game $\mathcal{G}$ is given by

$$
\Phi(\mathbf{a})=\sum_{j \in\{0\} \cup \mathcal{M}} \phi_{g}^{j}(\mathbf{a})+\frac{N_{a}}{2},
$$

where

$$
\phi_{g}^{j}(\mathbf{a})=\left\{\begin{array}{l}
\sum_{i \in C^{j}}\left(T_{s, i}+\frac{\left(O_{i}-1\right)}{D_{i}}\right)-\sum_{i \in C^{j}} I_{l, i}(n), j \in \mathcal{M}, \\
0, j=0 .
\end{array}\right.
$$

where $C^{j}$ is the set of all users that have selected the channel $j, O_{i}$ is the bonding order used on channel $j$, where $O_{i}$ can 


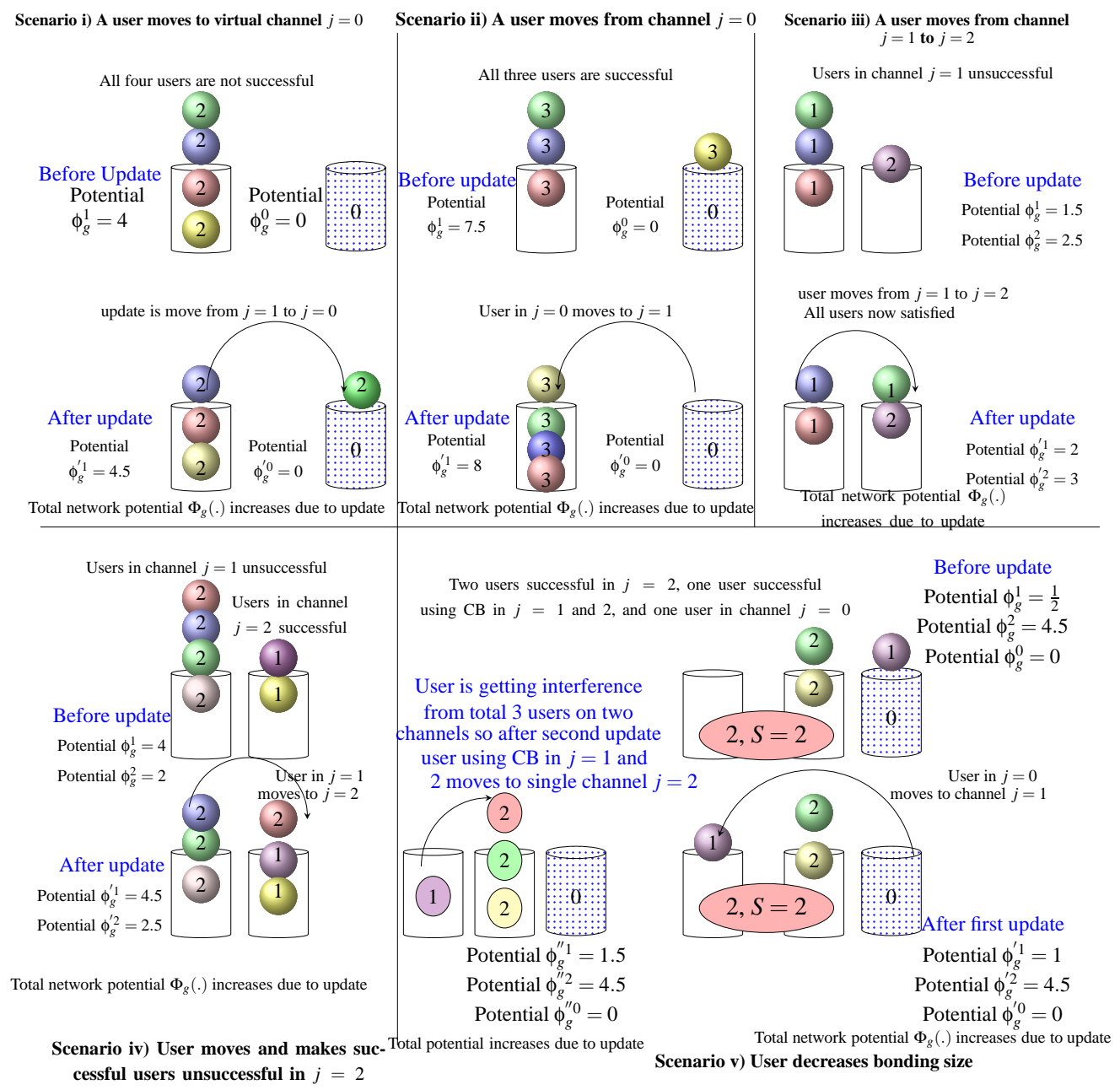

Fig. 4: Illustrative examples showing when the action update decisions for the proposed channel/bonding size selections increase utility then the ordinal potential function given in Eq. 13 is also increased. Each ball represents a user and each bin represents a channel. The number inside each ball represents a user's interference tolerance threshold $T_{s}, S=2$ means two channels are bonded. The factor $N_{a} / 2$ in Eq. 13 is added to each channel according to the number of active users present in that channel.

vary from 1 to $C_{m}$ (maximum bonding capability of user $i$ ) $O_{i}=1$ means user $i$ is using only one channel which is the $j$ th channel, or it is using more than one channel (channel bonding) and the $j$ th channel is the first channel out of the bonded channels, $O_{i}=2$ means user $i$ is using more than one channel and second channel is the $j$ th channel, and so on, $T_{S, i}$ is the tolerance threshold of user $i$ in terms of number of interfering users she can tolerate, $I_{l, i}(n)$ is the total number of edges (interference links) created by a user $i$ when it joins a channel or the total number of edges (interference links) removed when user $i$ leaves. It is a function of $n$ other users using that channel. Note that in the proposed ITE method we allow only one by one increase in channel size for $\mathrm{CB}$ (for details regarding how channels are selected/deselected please see Section IV-C). Finally, $N_{a}=N-\left|C^{j=0}\right|$ is the number of users that have not selected the null action. In Fig. 4, we also provide illustrative examples to help understand how the increase in utility corresponds to the increase in the generalized ordinal potential given in Eq. 13. We next show that the generalized ordinal potential function verifies the property in Eq. 12.
When a user $i$ 's demand is satisfied, i.e., $u_{i}=1$ in the current channel $j$, then she remains in the same action since she cannot increase her utility any further. We see that in this case no change in utility corresponds to no change in total potential. When a user's demand is not satisfied and the user does an action update by changing her action from $a_{i}$ to $a_{i}$ then $\Phi(\mathbf{a})>$ $\Phi(\mathbf{a})$ for all the following cases that can happen:

- 1) the user was using the virtual channel $j=0$, i.e., the null action, she finds an action that increases her $u_{i}>0$, she moves from action 0 to an action $j^{\prime} \neq 0$. The potential function value remains the same for the action 0 due to this move. The inner sum of the potential function corresponding to action $j^{\prime}$ (which is now used by user $i$ ) picks up an extra positive value, i.e., $\phi_{g}^{j^{\prime}}(\mathbf{a})-\phi_{g}^{j}(\mathbf{a})>0$. This is because the term $\phi_{g}^{j^{\prime}}(\mathbf{a})($ Eq. 14) either increases or remains the same and the number $N_{a}$ has increased by $\frac{1}{2}$.

- 2 ) the user was using action $a_{i} \neq 0$, i.e., not a null action, but is not successful in her usage, and is also not able to find a new action where she can be successful. The user selects the action $j=0$ (null action) to improve utility 
from $-c$ to 0 . Due to this move the potential function value remains the same for the virtual channel $j=0$. The inner sum of the potential function corresponding to action $j \neq 0$ (from which the user moved) picks up an extra positive value. This is because $\phi_{g}^{j \neq 0}(\mathbf{a})$ (Eq. 14) increases by at least 1 due to the decrease in the number of interfering edges connecting users, which is strictly greater than the decrease in $N_{a}$ by $\frac{1}{2}$. Hence the overall value is strictly positive. Thus, the increase in utility leads to increase in the potential.

- 3) the user was not using the null action and has $u_{i}=-c$, she finds a new action that increases her utility to $u_{i}>0$. The user moves to the new action. The inner sum of the potential function corresponding to the previous action (which was used by user $i$ ) picks up an extra positive value. This is because there is decrease in the number of interfering edges on previous selected channel from which the user moves. The inner sum of the potential function $\phi_{g}^{j^{\prime}}$ (á) (Eq. 14) corresponding to the new action (which is now used by user $i$ ) either remains the same or it increases. The number $N_{a}$ remains the same. Thus, the increase in utility leads to increase in the potential.

- 4) the user was not using the null action and has $u_{i}>$ 0 , she finds a new action which represents increase in bonding size non-contiguously and by using this action the user can further increase her $u_{i}$. The user selects the new action. Note that for the case where user has only contiguous $\mathrm{CB}$ capability this update was not possible. The inner sum of the potential function corresponding to the channels that are bonded under new action picks up an extra positive value. This is because the value $\frac{\left(O_{i}-1\right)}{D_{i}}$ increases. Thus, the increase in utility leads to increase in the potential.

- 5) the user is using an action $a_{i}$ which corresponds to bonded channels (either contiguous or non-contiguous), she finds thats her utility is decreased to $u_{i}=-c$ due to some other user moving to one of the bonded channels. It is important to note that when the other user moves and utility is decreased to $u_{i}=-c$ then it does not matter which bonded channel (contiguous or non-contiguous) the other user has moved to. Hence, the contiguous and the non-contiguous capabilities are equivalent in this case.

The user then finds that by reducing the bonding size she can increase her utility to $u_{i}>0$. The user selects this new action in which the bonding size is reduced. The inner sum of the potential function corresponding to the channels that the user was using picks an extra positive value as the number of interfering edges connecting users has decreased which is greater than the decrease in the value $\frac{\left(O_{i}-1\right)}{D_{i}}$. Thus, the increase in utility leads to increase in the potential.

Proposition IV.1. is an important and powerful result in this paper, for it implies that the channel/bonding selection is a potential game under undirected interference graph which in turn proves the existence of a NE. To the best of our knowledge, no other work has shown this property for channel/bonding selection.

The author of [6] has explicitly proved in [25], [26] this result for weakly acyclic games (please note that every potential game is a weakly acyclic game):

Theorem IV.1. ( [25], [26]) Let $G$ be a finite $N$-player weakly acyclic game in which all players use the Simple Experimentation dynamics with experimentation rate $\varepsilon>0$, then for all sufficiently small $\varepsilon$ a $N E$ is played at least $1-\varepsilon$ of the time.

The result given in [6] generalizes the above theorem for ITE. This generalization of the above theorem is also noted by another author in [27]. In fact, the author in [6] also clearly states that the principal difference between simple trial and error learning approach of [25], [26] and ITE in [6] is that the latter has an additional search phase that is triggered by decreases in payoff caused by someone else. This feature generalizes the results to hold also for any finite game with generic payoffs and at least one pure NE, such an equilibrium will be played a high proportion of the time provided that the experimentation probability is sufficiently small [6], [27].

Our result in Proposition IV. 1 has shown that the $N$-user channel/bonding size selection game (under the undirected interference graph representation) is a generalized ordinal potential game. As it is shown in [28] that every finite generalized ordinal potential game has at least one NE in pure strategies. We therefore can apply the ITE of [6] to our $N$ person generalized ordinal potential game $G$ and a pure NE will be visited at least $1-\varepsilon$ fraction of the times for any $\varepsilon>0$.

In the next section, we present our testbed implementation of the proposed channel/bonding size selection empowered by the ITE learning rule.

\section{PRototype IMPLEMENTATION AND EXPERIMENTAL EVALUATION}

\section{A. Modifications in WARPLab Research Framework}

We implemented the prototype by modifying the WARPLab, which is a research implementation framework for wireless communications algorithms and was originally presented in [5]. The WARPLab enables rapid implementation of physical layer algorithms using Xilinx Tools, such as System Generator for DSP [29], C programming, and MATLAB. It allows over-the-air (OTA) transmission of data packets using WARP boards. The main components of a WARP board are a Xilinx Virtex-family Pro FPGA, embedded processor, multiple connected radio boards, and ethernet connection to a host PC to also allow Matlab-based processing of transmit/received signals.

Our implementation is not straight forward use of original WARPLab presented in [5] as existing WARPLab framework does not support CB capabilities in WARP nodes. To provide the WARP nodes with CB capabilities we have modified the original WARPLab framework by implementing and incorporating a polyphase filter bank channelizer [30] on both transmit and receive sides of the WARPLab design. Moreover, to provide WARP nodes the capability to make intelligent $\mathrm{CB}$ decisions we have also implemented and incorporated the 
channel/bonding selection module using the ITE rule in the WARPLab design. In Fig. 1, we present a block diagram for main implemented functions on transmit/receive side of the WARP nodes for prototyping of the proposed method. The prototype implementation is comprised of three main components: 1) OFDM-based transmitter and receiver functions that enable real OTA wireless communication of data packets. Complete details of these functions and codes can be found in [31]. 2) Implementation of Channelizer module in WARPLab by us to allow the transmitter/receiver to perform CB for real OTA wireless communications. 3) Implementation of ITE-based CB selection module by us in WARPLab framework.

\section{B. OFDM-based transmitter/receiver functions}

On the transmitter side the prototyped system performs the following main functions before processed data is given as input to the channelizer module after which the I/Q data is sent to the RF front end for OTA transmissions of data packets (see Fig. 1): 1) Random data generation; 2) modulation of generated random data to complex constellation symbols; 3) insertion of pilot values; 4) inverse fast Fourier transform of the matrix of data and pilot values; 5) insertion of cyclic prefix and reshape to a vector; 6) preamble insertion to construct full time-domain OFDM waveform; 7) Interpolation and input to the channelizer module.

On the receiver side the following main functions are performed after I/Q samples are received from the RF front end and are processed by the implemented channelizer (see Fig. 1): 1) Long training sequence (LTS) correlation for synchronization. When no valid correlation peak is found then the process is stopped and unsuccessful communication is declared, otherwise the process is moved to the next function for further processing; 2) carrier frequency offset (CFO) correction and cyclic prefix removal; 3) fast Fourier transform; 4) channel estimation from frequency-domain LTS and residual phase error estimation from frequency-domain pilot tones; 5) equalization of subcarriers containing data using channel and phase error estimates; 6) complex symbol demodulation to data values; 7) update of user mental state (mood) in the ITE module based on successful/unsuccessful reception of data.

\section{Channelizer Module to enable CB capability in WARP nodes}

A channelizer takes a wide bandwidth signal and transforms it into several narrow bandwidth signals. The implemented channelizer has a polyphase filter bank structure and is based on the model presented by Xilinx in [30]. In Fig. 1, our presented block diagram also shows implemented main functions (green color boxes) of the channelizer module on both transmit and receive sides. The channelizer enables a WARP node to be a resource efficient multichannel digital transmitter or receiver. Taking into account the ITE-based CB selection, the channelizer adaptively selects a filter with particular number of coefficients. For example, consider the case that there are $M$ available channels each of bandwidth $W$, and a user using ITEbased CB method decides to bond two channels, i.e., $S_{i}=2$. This is achieved by using a channelizer that takes the total
$M W$ bandwidth, channelizes it into multiple narrow channels each of bandwith $2 W$, and the user makes selection from one of these available bonding selections.

For efficiency and simplicity, we have performed the implementation in a way that the same channelizer can be used to channelize the total bandwidth $M W$ into narrow channels of any desired bandwidth $k W$. This is achieved by performing the following functions on the transmitter side: 1) Polyphase filtering where the channelizer's prototype polyphase filter can use variable number of coefficients to generate multiple channels of a particular $k W$ bandwidth. Depending on the bonding size selection, a filter with a particular suitable number of coefficients is created; 2) IFFT is performed on the data from the interpolation module (see Fig. 1) of the transmitter side; 3) finite impulse response (FIR) polyphase filter is setup to process in single instance the selected data channel and also other non-data channels; 4) the data is reshaped into single stream and sent to the RF front end. The receiver side involves the following main functions: 1) The channelizer reshapes received multiple channels samples; 2) it performs FIR polyphase filtering; 3) IFFT on the received samples; and 4) extract samples from the data channel and forwards it to the LTS/correlation block (see Fig. 1).

\section{Evaluation of the Implemented Prototype with OTA Exper- iments}

In this subsection, we evaluate the performance of the implemented prototype on the WARP nodes. In the evaluation setup, the WARP nodes were transmitting/receiving OTA data packets in $2.4 \mathrm{GHz}$ unlicensed band and were connected to a laptop computer via ethernet port. Setup for OTA experiments to test the performance of implemented method is also illustrated in Fig. 5. The evaluation results were recorded using the Matlab software. A wireless system of two Warp nodes were transmitting/receiving OTA OFDM signals. For the experimental testing the $\mathrm{CB}$ capability of the two nodes were set to $C_{i}=2$, i.e., the two nodes could either use a single channel or a bonded channel of size two to communicate OTA data packets. There were a total of $M=11$ single channels of bandwidth $W$ and 5 bonded channels of $2 W$ bandwidth made available to the WARP nodes through the implemented channelizer. In Table I, we present some of the main parameters relating to the OTA experiments. All OTA experiments were performed in the laboratory room where there were RF signals from other WiFi users/access points present.

In Fig. 6, we present the performance of the implemented method in terms of its accuracy and convergence towards finding those channel/bonding size selection that allows the WARP nodes to successfully communicate data packets. The results in Fig. 6 are plotted by averaging the payoff values obtained via multiple rounds of OTA experiments, where in each round the WARP nodes perform channel/bonding selections and communications for 200 time steps. To obtain the performance in terms of finding channels that lead to successful communications, we also created synthetic interference in multiple channels in a way that each channel 


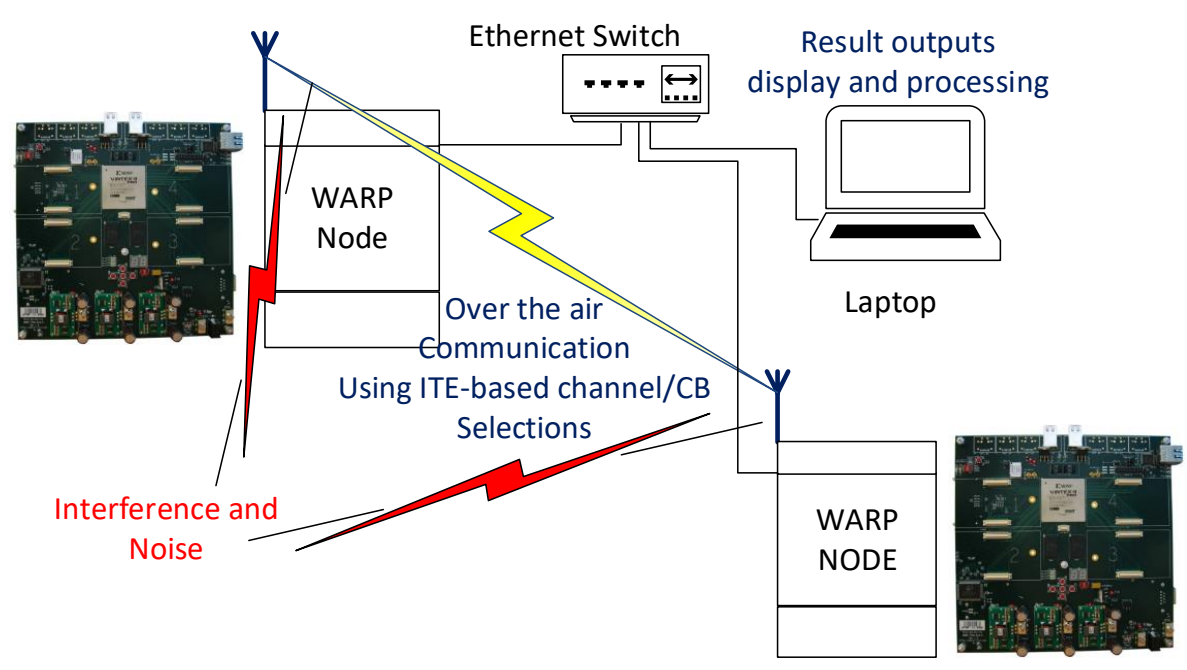

Fig. 5: OTA measurements setup for the testing of the implemented prototype.

TABLE I: Some example parameters used during OTA Experiments.

\begin{tabular}{|c|c|}
\hline Frequency band & $2.4 \mathrm{GHz}$ (unlicensed) \\
\hline Number of single channels (using the channelizer) & 11 \\
\hline Number of bonded channels of size 2 (using the channelizer) & 5 \\
\hline Single Channel Vector ( 1 means interference in the channel is below the threshold, 0 means above) & {$\left[\begin{array}{lllllllllll}1 & 1 & 0 & 0 & 0 & 0 & 0 & 0 & 0 & 0 & 0\end{array}\right]$} \\
\hline Bonded Channel Vector ( 1 means interference in the channel is below the threshold, 0 means above) & {$\left[\begin{array}{lllll}1 & 0 & 0 & 0 & 0\end{array}\right]$} \\
\hline Modulation Order & QPSK \\
\hline Number of OFDM Subcarriers & 64 \\
\hline Number of Data Subcarriers & 48 \\
\hline Cyclic Prefix Length & 16 \\
\hline Number of OFDM Symbols & 2 \\
\hline Number of Data Symbols & $2 \times 48$ \\
\hline Interpolation Rate & 0.8 \\
\hline FFT offset & 4 \\
\hline Number of Samples & $2^{20}$ \\
\hline Sampling Frequency & $40 \mathrm{MHz}$ \\
\hline
\end{tabular}

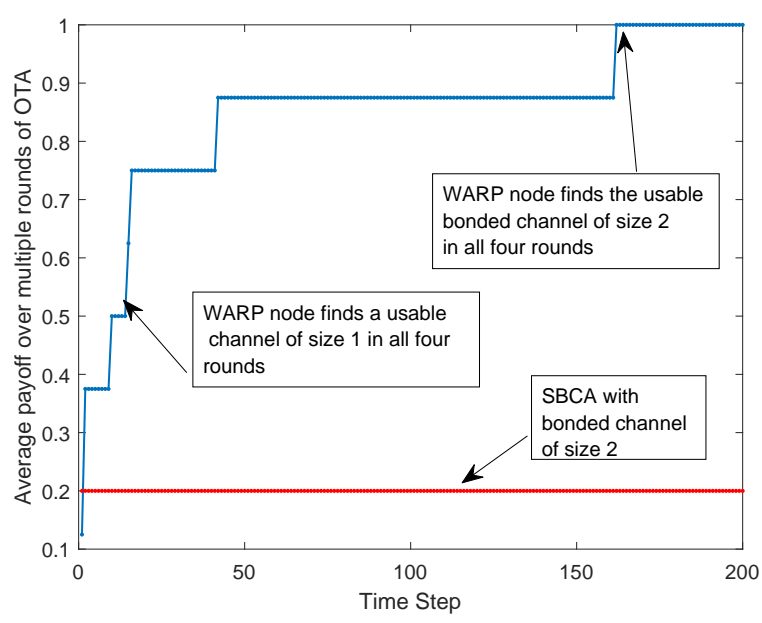

Fig. 6: Average payoff as a function of time steps for the OTA experiments.

had different interference levels. For example, interference on channels was created so that there was only 1 channel out of 5 total bonded channels (with bandwidth size $2 W$ ) that satisfied the interference threshold level for the receiver side, and there were 2 channels out of 11 total single channels (with bandwidth size $W$ ) that satisfied the threshold level. This means that for the single channel selection case only 2 channels of bandwidth size $W$ were suitable for the WARP nodes to communicate on, and for the bonding case only 1 channel was suitable for communications. When the WARP nodes selected an unsuitable channel for communication then it encountered interference above the interference tolerance level which resulted in unsuccessful communications. The nodes update the channel selection based on observed packet successes and failures. It can be seen from Fig. 6 that using the implemented ITE-based method WARP nodes obtained average payoff of 0.5 or more in no more than 20 time steps, and obtained average maximum payoff of 1 in no more than 165 time steps. In other words, in no more than 20 time steps the WARP nodes were always able to find and use a single usable channel to obtain an average pay off of 0.5 , and in no more than 165 time steps they were always able to find the usable bonded channel to obtain the average maximum payoff of 1 .

SBCA uses fixed bonding size and it does not perform adaptations. When it is set to use bandwidth size $2 W$ then in multiple rounds of 200 time steps its average obtained payoff is 0.2 . This is due to the reason that 1 out of 5 channels of bonding size 2 are usable. When it is set to use bandwidth 
TABLE II: Some example parameters used during simulations.

\begin{tabular}{|c|c|}
\hline Parameters & Values \\
\hline Site radius $N_{R}$ & $50 \mathrm{~m}$ \\
\hline Minimum distance between transmitter and receiver & $8 \mathrm{~m}$ \\
\hline Maximum distance between transmitter and receiver & $30 \mathrm{~m}$ \\
\hline Frequency band & $2.4 \mathrm{GHz}$ (unlicensed) \\
\hline Number of channels & $0 \%$ and $5 \%$ \\
\hline Adjacent Channel Interference (ACI) & $20 \mathrm{~mW}$ \\
\hline Maximum transmission power & $1 \mathrm{dBi}$ \\
\hline Transmitter and receiver antenna gain & $5 \mathrm{~cm}$ \\
\hline Transmitter and receiver antenna length & 3 \\
\hline Path-loss exponent $\alpha$ & $5 \mathrm{~dB}$ \\
\hline SINR threshold $\gamma_{0}$ & 0.05 \\
\hline$\phi_{b}$ (Equation 10$)$ & 0.02 \\
\hline Explore parameter $\varepsilon$ & 1000 \\
\hline Simulation iterations & $3000,6000,8000$ \\
\hline Time slots per iteration & $\mathbf{C}_{\mathbf{B}}=[3,3,3,2,2,1,1,1], \mathbf{C}_{\mathbf{B}}=[3,3,3,2,2,2,2,2]$ \\
\hline User CB demand vector & \\
\hline User rate demand vector & {$[22.5,19.2,17.8,33.8,33.1,18.4,34.5,27.6]$ Mbps } \\
\hline
\end{tabular}

size $W$ then in multiple rounds of 200 time steps its average obtained payoff is 0.09 . This is due to the reason that 2 out of 11 single channels are usable but a single channel gives a payoff of only 0.5 which gives $(2 / 11) \times 0.5=0.09$. The DCBA method also does not performs adaptations but it can use one channel ( $W$ bandwidth) when both channels $(2 W$ bandwidth) are not available but only one of them is available for usage. As a result it can perform slightly better than the SBCA method. It is easy to see that due to lack of adaptations in channel/bonding size selections both the SBCA and DBCA methods cannot achieve the average maximum payoff of 1 which makes their performance inferior to the proposed ITEbased method.

\section{Performance Evaluation using Numerical ANALYSIS}

\section{A. Performance metrics, and simulation parameters}

Using numerical analysis, we evaluate the proposed method in terms of probability of being in NE, average user payoff, average sum data rate of all the users, user blocking rate, and average CB size utilized by a user. In our results, we present average sum data rates as a function of time step $t$. Sum data rate in each step is calculated based on the Shannon capacity formula:

$$
\tau_{\text {sum }}=\sum_{i=1}^{N} S_{i} W \log _{2}\left(1+\gamma_{i}\right)
$$

where $S_{i}$ is the utilized bonding size of user $i, W$ is the bandwidth of a channel, and $\gamma_{i}$ is the SINR of user $i$ given by Eq. 7. Average sum data rate results are plotted by obtaining averages using simulations running over several Monte Carlo runs and in each Monte Carlo run calculations are done using Eq. 15. For the same given fixed number $M$ of available channels, it can happen that as the number of users and/or their bonding capability increases users experience higher levels of interference. As a result some users may be unable to communicate on any channels due to SINR outage at their respective receivers. We consider blocking incurred by a user to be the ratio of the average number of blocked users to the total number of users:

$$
R_{b}=\frac{\bar{N}_{b}}{N}
$$

We also consider non-satisfaction incurred by a user which is given by

$$
R_{u}=\frac{\bar{N}_{u}}{N}
$$

where $\bar{N}_{u}$ is the average number of users that have utility greater than 0 but less than maximum utility of 1 . Nonsatisfaction means that a user can successfully communicate but cannot use successfully her maximum desired CB size. Our results also evaluate the impact of $\mathrm{ACI}$ on the performance of the proposed technique. We also compare the proposed solution with a centralized CB selection method in which a centralized entity finds a channel/CB assignment for all users in the network that maximizes the total sum payoff. Note that the centralized method knows all users' $\mathrm{CB}$ capabilities, and the interference between users in any channel/CB assignment. Using this knowledge it performs an exhaustive search over a set of all possible channel/bonding selections to find optimal selections. In practice, the centralized method is computationally highly intensive due to combinatorial explosion and is not suitable for distributed and uncoordinated channel/CB selection scenarios considered in this work. It is only used to establish a baseline for comparing the results obtained from the proposed distributed method. For example, the optimal solution obtained via centralized solution can help us in evaluating the quality of the $\mathrm{NE}$ outcome that has been reached via the proposed method under the same scenario. We also provide comparison results with a typical explore/exploit method which we have adapted to CB. The idea behind this method can be summarized as follows: a user is either in an exploit state, in which it will select the same channel/CB selection with a probability that is a function of the SINR (channel quality metric), or in an explore state, where it will explore a new CB selection. We also compare the performance of the proposed method with the two methods that are currently being used to support $\mathrm{CB}$ in unlicensed channels: SBCA and DBCA [1].

In Table II, we present the main simulation parameters used to evaluate and compare the performance of the proposed method under different scenarios. 


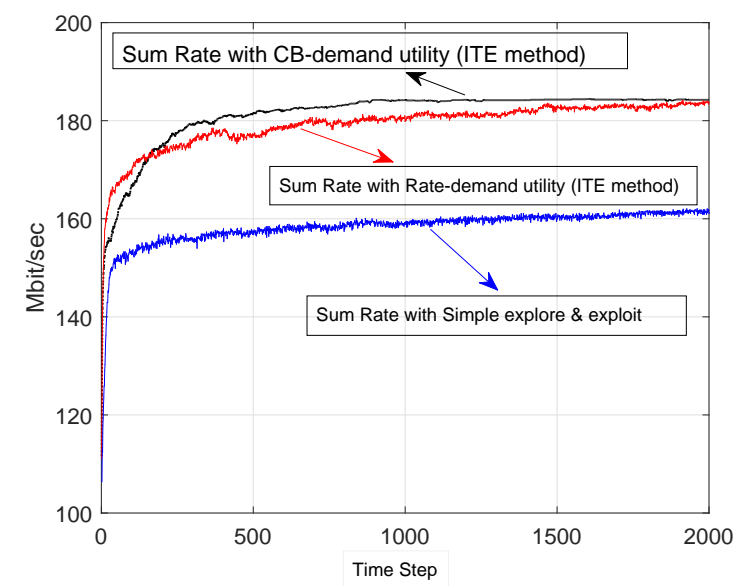

Fig. 7: Average sum rates as a function of time step for ITE with CB demand based utility and Rate demand based utility, and for simple explore/exploit with CB demand based utility.

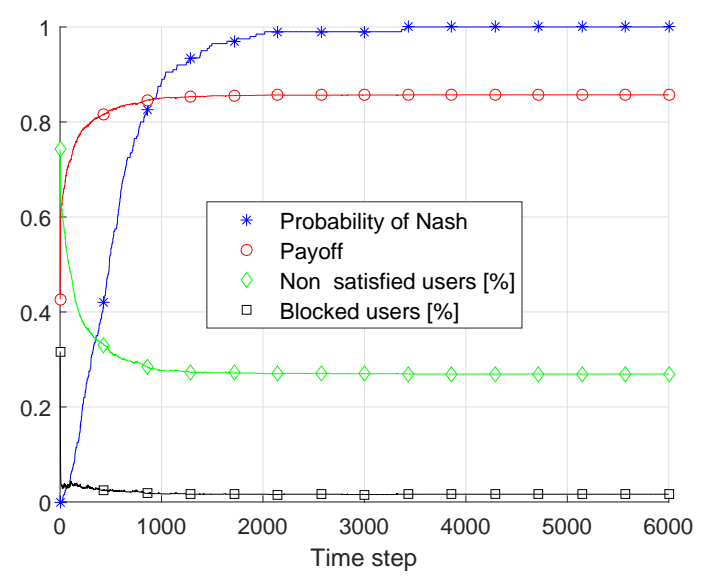

(a)

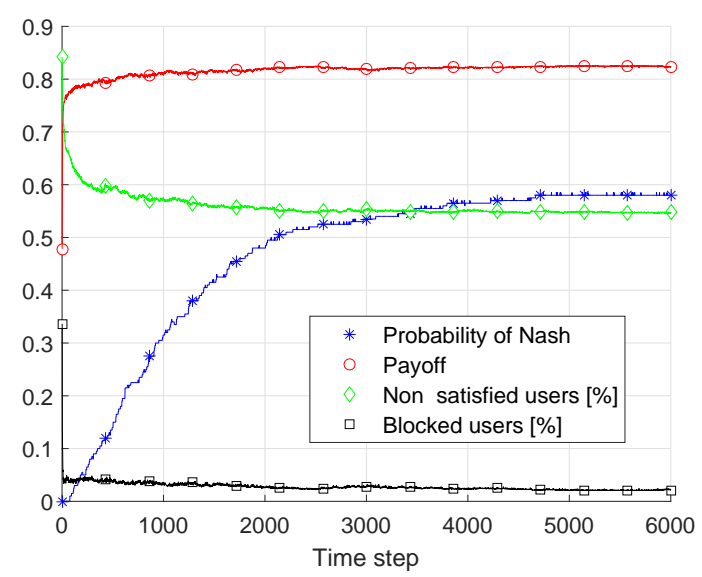

(b)

Fig. 8: Probability of being in NE, average payoff, percentage of users not satisfied, and percentage of users blocked as a function of time step for a) CB demand based utility; and b) Rate demand based utility.
B. Comparison of the CB-demand based utility with the Rate demand utility

The rate-demand based utility function which integrates the $R_{i}$ is given by:

$$
u_{i}= \begin{cases}\min \left(\left[\frac{S_{i} R_{i}}{R_{D, i}}, 1\right]\right), & \gamma_{i} \geq \gamma_{0}, a_{i} \in \Sigma_{i} \\ -c, & \gamma_{i}<\gamma_{o}, a_{i} \in \Sigma_{i} \\ 0, & a_{i}=0 .\end{cases}
$$

where $S_{i}$ represents the number of channels utilized by a user $i$ ( $S_{i}$ is limited by the maximum bonding capability $C_{i}$ of the user $i$ ). Each user $i$ has a rate demand $R_{D, i}$. A user achieves a utility of 1 when she is able to successfully achieve the desired rate. Note that success means: a user selects an action $a_{i} \in \Sigma_{i}$ that corresponds to some bonding size, and using this action her communication to her intended receiver is successful, i.e., received SINR $\gamma_{i}$ is greater than a defined threshold SINR $\gamma_{0}$. When she is able to successfully communicate but the rate is less than the required demanded rate then she achieves a utility proportional to the ratio of the achieved rate to the demanded rate. When the user is not successful then the utility is $-c$, where $0<c \ll 1$ is the penalty of transmitting (wasting power) despite the fact that the link cannot satisfy the quality of service requirement of the user's applications, and the utility is zero when the user selects the null action. Note that a user seeks to increase her received utility $u_{i}$ only when its less than 1.

In Fig. 7 and Figs. 8a-b, we compare the results obtained via $\mathrm{CB}$ demand and rate demand based utility functions. The figures show that under similar network parameters for both the rate demand and the $\mathrm{CB}$ demand based utility functions the proposed ITE-based method performs equally well in terms of achieved average sum rate, average percentage of blocked users and average payoff. However, the CB demand utility performs better in terms of probability of being in NE and also in terms of average percentage of non-satisfied users. The reason for this is that the rate demand based utility function is sensitive to small changes in the achieved rate. This can make difficult for the users using rate demand based utility function to achieve NE and also difficult to be satisfied (to get their target rate). To conclude, using rate based utility instead of CB size based utility does not lead to significant rate benefits. In Fig. 7, we also compare the results obtained with sum rate performance of simple explore and exploit method. The figures show that under similar network parameters the ITE-based method outperforms the simple explore and exploit method in terms of sum rate performance.

\section{Probability of being in a NE and comparison with simple explorelexploit-based method}

In Fig. 9, the probability of being in an NE at time step $t$ for the proposed ITE-based method with and without channel quality measurements (see Section IV-C) are shown for various $\mathrm{CB}$ and network scenarios. The results are obtained by simulating 200 random network instances, where in each instance the ITE method is played for 8,000 time steps. Fig. 9 presents results for the scenarios where users have 


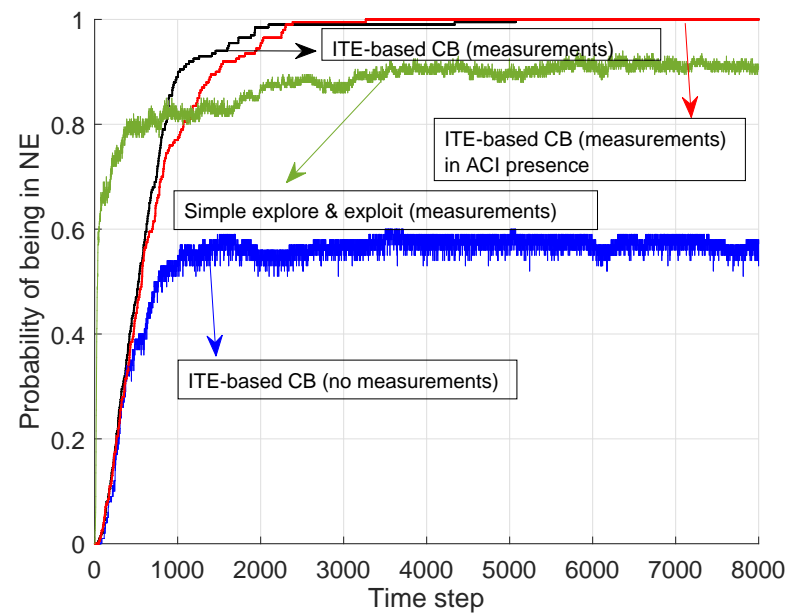

Fig. 9: Probability of Being in NE as a function of time steps.

heterogeneous $\mathrm{CB}$ demands. We evaluate the performance when there are $M=8$ channels, and the $\mathrm{CB}$ demand vector for $N=8$ users is $\mathbf{C}_{\mathbf{B}}=[3,3,3,2,2,1,1,1]$. It can be seen from the figure that the number of time steps taken for the network to be in a NE with very high probability is significantly improved when the users explore using channel quality measurements in the proposed ITE method. It can be seen from Fig. 9 that when no measurements are utilized during the exploration phase then the probability of being in NE is no more than 0.6. However, due to the random experiments from time to time the network will still converge to NE but it may take longer time. Figure 9 shows that with measurements this probability is increased to 1. In Fig. 9, we also evaluate the impact of ACI on the proposed ITE method. It can be seen that the presence of ACI slightly degrades the performance of the proposed ITE method, however, it can be seen that the network still converges to NE probability of 1. In Fig. 9, we also compare the performance of the ITE-based method with simple explore and exploit method (with measurements). It can be seen from the figure that ITE-based method outperforms the simple explore and exploit method in terms of probability of being in NE. Moreover, the results in the figure also show that the simple explore and exploit method is rather unstable as results seem to continuously fluctuate.

\section{Changes in $C B$ demands}

Fig. 10 presents results for the scenarios where users have heterogeneous CB demands and they can change at some time step. We evaluate the performance when there are $M=8$ channels, and the $\mathrm{CB}$ demand vector for $N=8$ users is initially $\mathbf{C}_{\mathbf{B}}=[3,3,3,2,2,1,1,1]$ and at the time step 3000 it changes to $\mathbf{C}_{\mathbf{B}}=[3,3,3,2,2,2,2,2]$. This means that each of the three users which demand one channel, at time step 3000 onwards demand two channels each. It can be seen from the figure that after average payoff and a NE convergence, when bonding size demand of some users changes, the users starts a search for a new action, eventually settling on actions with a probability that increases monotonically with their realized payoffs.

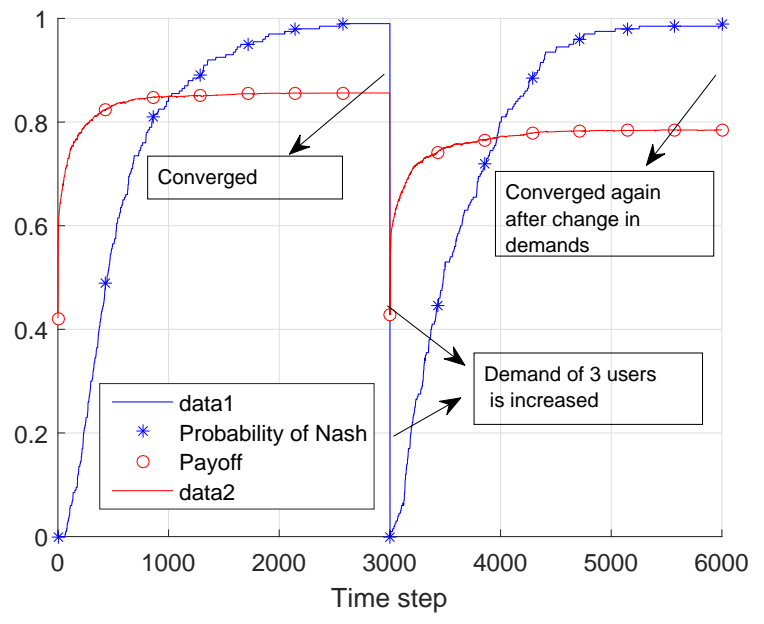

Fig. 10: Probability of Being in NE and average per user payoff as a function of time steps for the case where $\mathrm{CB}$ demands of some users change at time step 3000 .

TABLE III: Results showing POA and POS for various scenarios. $M=8$ single channels and $\mathbf{C}_{\mathbf{B}}=[3,3,3,2,2,1,1,1]$.

\begin{tabular}{|l||l||l|}
\hline Scenario & POS & POA \\
\hline Site Radius $N_{R}=50 \mathrm{~m}, A C I=0$ & 0.9748 & 0.8696 \\
\hline Site Radius $N_{R}=50 \mathrm{~m}, A C I=0.05$ & 0.9704 & 0.8363 \\
\hline Site Radius $N_{R}=80 \mathrm{~m}, A C I=0$ & 0.9905 & 0.8201 \\
\hline Site Radius $N_{R}=80 \mathrm{~m}, A C I=0.05$ & 0.9805 & 0.8014 \\
\hline \hline
\end{tabular}

\section{E. Efficiency of NE in terms of POA and POS}

Since the channel/bonding selection game may have many NEs, we also measure how efficient a NE obtained from the proposed method is in the game. We have at least two natural measures, corresponding to the best and the worst NE. In game theory, the price of anarchy (POA) and the price of stability (POS) are used to evaluate the efficiency of the worst and the best NE. The POS and the POA are given, respectively, by

$$
\mathrm{POS}=\frac{\text { value of best NE payoff }}{\text { value of optimal solution payoff }},
$$

and

$$
\text { POA }=\frac{\text { value of worst NE payoff }}{\text { value of optimal solution payoff }} .
$$

In Table III, we evaluate the proposed method in terms of POA and POS for different scenarios. We present results using two different network site radii of $N_{R}=50 \mathrm{~m}$ and $N_{R}=80 \mathrm{~m}$, and also consider scenarios with and without ACI. Results for each particular scenario are obtained using the same multiple random network instances, where for each network instance the game is repeated several times, and in each repetition the game is played for 5000 time steps. Presented results are for the scenarios where users have heterogeneous $\mathrm{CB}$ demands. We evaluate the performance when there are $M=8$ channels, and the $\mathrm{CB}$ demand vector for $N=8$ users is $\mathbf{C}_{\mathbf{B}}=[3,3,3,2,2,1,1,1]$. It can be seen from the results in Table III that the POA is no less than 0.8. This means that there is no more than $20 \%$ degradation due to distributed decisions of users under the proposed method as compared to the centralized optimal solution. Moreover, POS is close to 


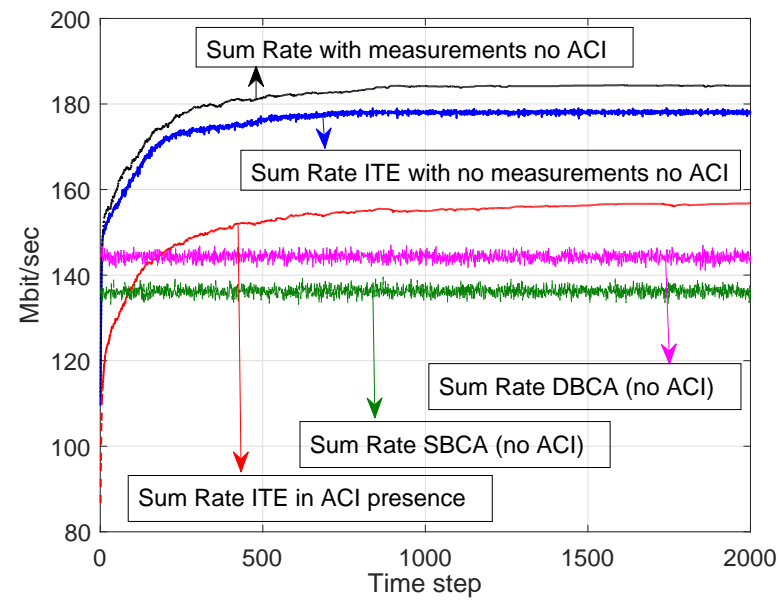

Fig. 11: Average sum rates as a function of time steps.

TABLE IV: Results showing obtained average payoff per user using the centralized solution, and the proposed distributed method for $C_{B}=$ $[3,3,3,2,2,1,1,1]$.

\begin{tabular}{|l||l||l|}
\hline Scenario & Centralized & Proposed \\
\hline$N_{R}=50 \mathrm{~m}, A C I=0$, No measurements & 0.915 & 0.80 \\
\hline$N_{R}=50 \mathrm{~m}, A C I=0$, Measurements & 0.915 & 0.85 \\
\hline$N_{R}=50 \mathrm{~m}, A C I=0.05$, Measurements & 0.89 & 0.795 \\
\hline$N_{R}=80 \mathrm{~m}, A C I=0$, Measurements & 0.985 & 0.915 \\
\hline
\end{tabular}

0.97 which means that the best NE payoff per user is close to the optimal centralized solution (that maximizes the average payoff per user).

\section{F. Comparison with the SBCA, the DBCA, and the centralized methods}

In Figs. 11, we present a comparison of the average sum data rates achieved by the proposed method (with and without channel quality measurements) to the average sum data rates achieved using the SBCA and DBCA methods. In Fig. 11, for $M=8$ channels, the $\mathrm{CB}$ demand vector for $N=8$ users is $\mathbf{C}_{\mathbf{B}}=[3,3,3,2,2,1,1,1]$. The results in Fig. 11 show that the proposed method achieves higher sum rates when the users explore using channel quality measurements. It can be also seen that the presence of ACI degrades the sum rate performance. Moreover, the highest sum rates are obtained when the proposed method is used as compared to the SBCA and the DBCA methods. The reason for this is as follows. The SBCA and the DBCA methods do not utilize learning-based adaptation in their channel/bonding size selections, whereas the proposed method utilizes adaptations in channel/bonding size selections that take into account the channel quality metric based on SINR. The proposed method enables users to select those bonding sizes that increase the likelihood of achieving higher data rates.

In Fig. 12a, we evaluate the performance of the proposed method in terms of three different performance metrics. It can be seen from Fig. 12a that for the proposed method the average payoff per user is 0.85 while for the same scenarios the achieved payoff in Figs. $12 \mathrm{~b}$ and $12 \mathrm{c}$ under the SBCA and the DBCA methods is only 0.58 and 0.6 , respectively.

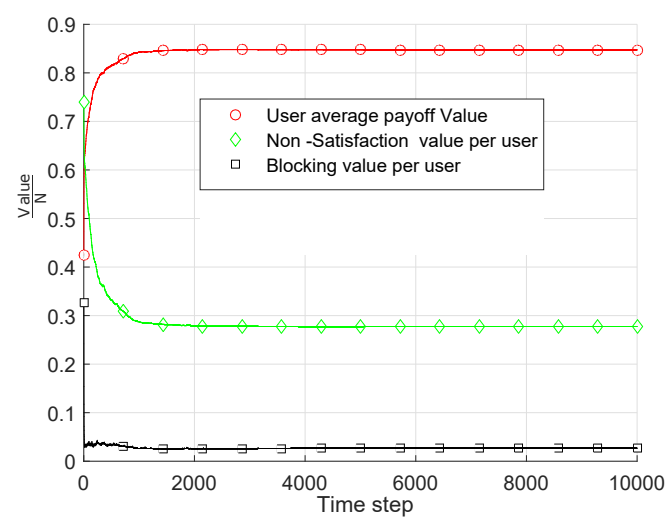

(a)

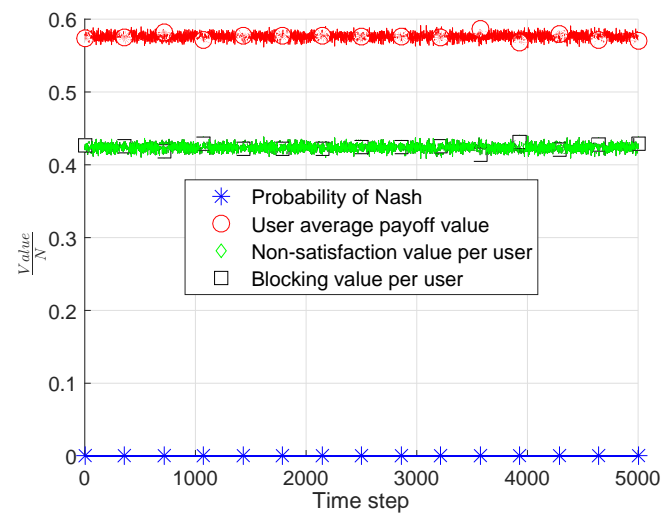

(b)

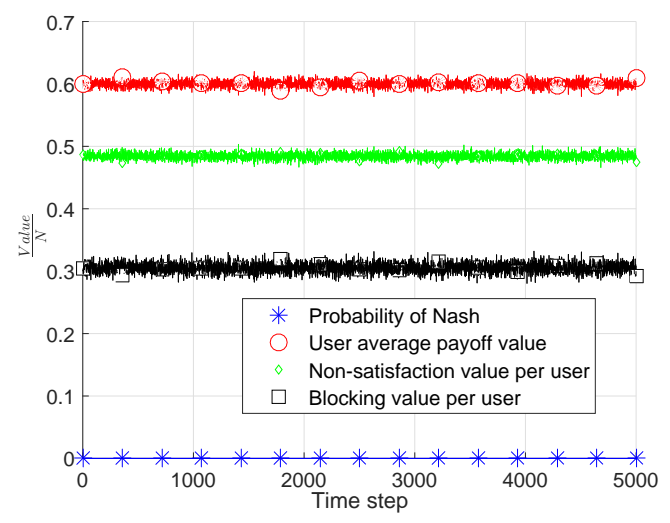

(c)

Fig. 12: $\frac{\text { Value }}{N}$ as a function of time steps, where $\frac{\text { Value }}{N}$ is used to represent average payoff per user, average blocking incurred per user, and average non-satisfaction incurred per user. Number of users is $N=8$ and the CB demand vector is $\mathbf{C}_{\mathbf{B}}=[3,3,3,2,2,1,1,1]$. 
The average blocking incurred by a user using the proposed method is only 0.02 while it is 0.42 and 0.3 for the SBCA and DBCA methods, respectively. Finally, average non-satisfaction incurred by a user using the proposed method is close to 0.28 while for the SBCA and the DBCA methods it is 0.42 and 0.5 , respectively. The three figures show that for all three performance metrics, the proposed method outperforms the SBCA and the DBCA methods. Moreover, it can be also seen from Figs. $12 b$ and $12 c$ that the probability of being in NE for the SBCA and the DBCA is 0, whereas, in Sections VI-B and VI-C, we have extensively evaluated the performance of the proposed method and have shown that the probability of being in NE is close to 1 for the proposed method.

In Table IV, we compare the average payoff per user performance of the proposed method with the centralized solution under four different scenarios. It can be seen that when no channel quality measurements are utilized then there is an average difference of 0.115 between the average payoff obtained via centralized solution and the proposed distributed method. However, channel quality measurements reduce this difference in average payoffs to 0.0615 . It can be seen that when ACI is taken into account then the performance in terms of average payoff per user of the proposed method degrades slightly more as compared to the centralized solution. It can be also seen that due to ACI the difference in average payoffs is 0.095 . Table IV also shows that when the network site radius $N_{R}$ is increased to $80 \mathrm{~m}$ as compared to $N_{R}=50 \mathrm{~m}$ then the centralized solution on average reaches close to the maximum possible payoff value of 1 per user. At the same time, the performance of the proposed method is also increased to the average value of 0.915 per user.

\section{G. Non-contiguous $C B$ selections}

In terms of performance metrics, such as sum rate, blocking rate, and probability of being in a NE, in Figs. 13a-b, we present the performance of the proposed method under noncontiguous bonding selections. The performance is also compared with the case where the users are limited to contiguous $\mathrm{CB}$. It can be seen in the figures that for the case where $A C I=0$, non-contiguous bonding can achieve either the same performance or slightly better performance as compared to contiguous bonding in terms of average sum rate, average payoff per user, percentage of users blocked, percentage of non satisfied users. However, it can be also seen that the non-contiguous bonding takes longer time to reach probability of being in NE. This is due to the reason that the noncontiguous bonding increases the number of ways a user can bond channels and distributed learning typically takes more time to reach a NE when there are more possible combinations (combinatorial explosion). We also evaluate the average sum rate performance and the impact of adjacent channel interference (ACI) on its performance under the noncontiguous CB. Fig. 14 shows that when the ACI is set to $5 \%$ then the performance of non-contiguous $\mathrm{CB}$ performance slightly degrades in terms of average sum rate.

Our results show that for the proposed method the network remains in a NE with very high probability most of the time.

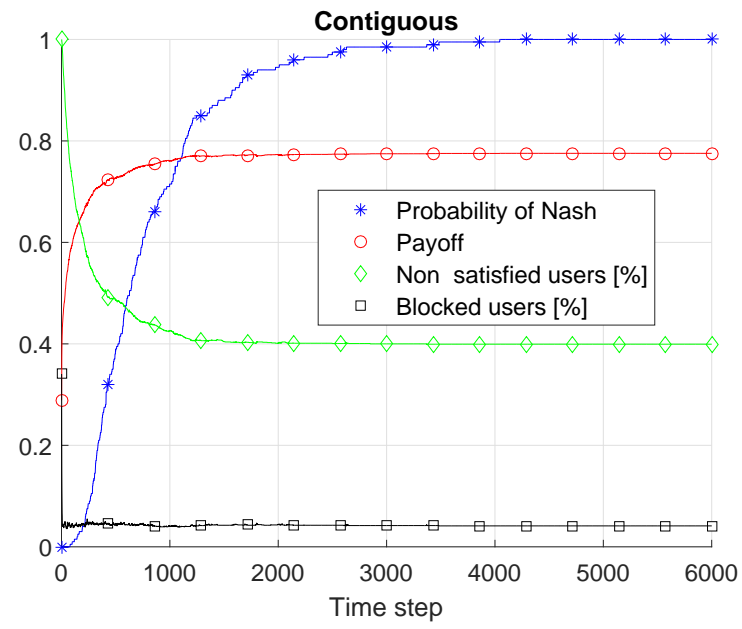

(a)

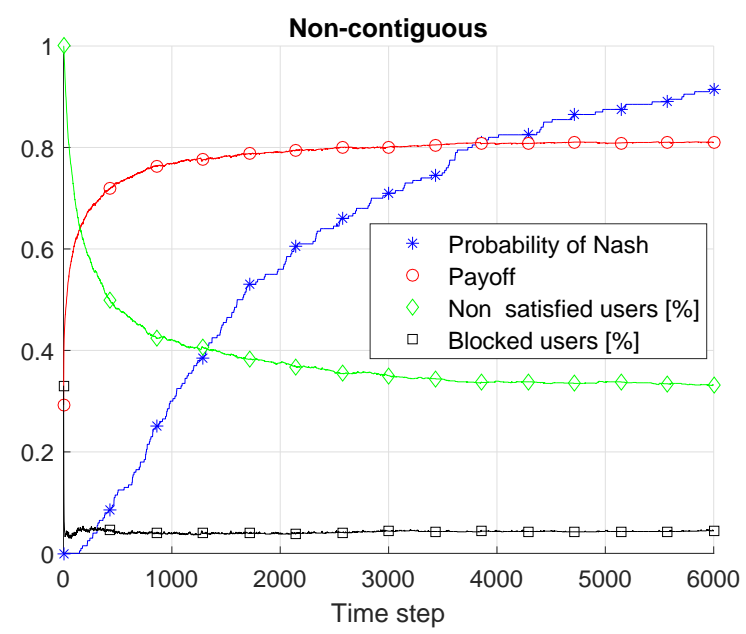

(b)

Fig. 13: Probability of being in NE, average payoff, percentage of users not satisfied, and percentage of users blocked as a function of time step for $\kappa=1$ under a) contiguous $\mathrm{CB}$; and b) non-contiguous $\mathrm{CB}$. User rate demand vector $R_{D}=$ $[22.5,19.2,17.8,33.8,33.1,18.4,34.5,27.6]$ Mbps. After performing 1000 simulation iterations for 6000 time slots and calculating average achievable rates the vector $R_{D}$ was set.

This means thats the users in the network spend most of the time in the content state. The proposed method requires very little measurements because experimentation probability $\varepsilon$ is 0.02 when in content state. This is one important feature of ITE-based methods as even with a small probability of experimentation it can implement NE behavior [6]. In Table V, we present results which show that under $C_{B}=[3,3,3,2,2,1,1,1]$ for $\varepsilon=0.02$ average measurements per time step are only 0.0059 and in total there has been 17.6 average number of measurements for a total of 3000 time steps. Moreover, when the users CB demand is increased to $C_{B}=[3,3,3,2,2,2,2,2]$ the total number of measurements are only 57 for $\varepsilon=0.02$. Our results in the same table also show that when we set $\varepsilon=1$ (always measure in content state) then there is almost no improvement in the average payoff. If we introduce a penalty cost per measurement $c_{m}$ in the average payoff per user given 


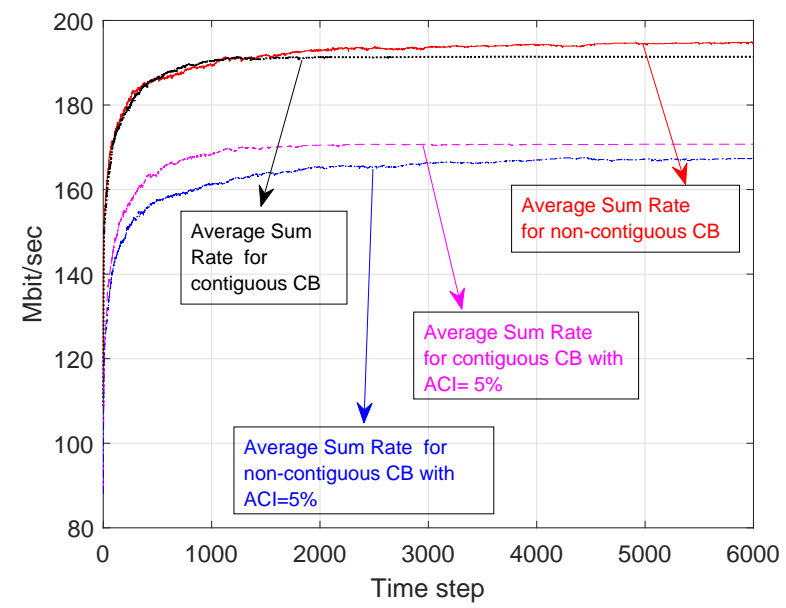

Fig. 14: Average sum rates as a function of time step for $\kappa=1$ under a) non-contiguous $\mathrm{CB}$ (with and without $\mathrm{ACI}$ ); and b) contiguous $\mathrm{CB}$ (with and without $\mathrm{ACI}$ ).

TABLE V: Results showing the impact of varying experiment probability $\varepsilon$ for $C_{B}=[3,3,3,2,2,1,1,1]$ and $C_{B}=[3,3,3,3,2,2,2,2]$, respectively.

\begin{tabular}{|l||l||l|}
\hline Experiment probability & $\varepsilon=1$ & $\varepsilon=0.02$ \\
\hline$E\left[u_{i}\right]$ (average payoff/time step) & 0.855 & 0.841 \\
\hline$E\left[N_{m}\right]$ (average measurements/time step) & 0.2649 & 0.0059 \\
\hline$\psi_{i}$ (payoff with cost)(with $\left.c_{m}=1\right)$ & 0.5901 & 0.8351 \\
\hline$\psi_{i}$ (payoff with cost) (with $\left.c_{m}=0.2\right)$ & 0.8020 & 0.8398 \\
\hline$E\left[\hat{N}_{m}\right]$ (average total measurements) & 794 & 17.6 \\
\hline$E\left[u_{i}\right]$ (average payoff/time step) & 0.75 & 0.74 \\
\hline$E\left[N_{m}\right]$ (average measurements/time step) & 0.4521 & 0.0095 \\
\hline$\psi_{i}$ (payoff with cost)(with $\left.c_{m}=1\right)$ & 0.3 & 0.731 \\
\hline$\psi_{i}$ (payoff with cost)(with $\left.c_{m}=0.2\right)$ & 0.66 & 0.738 \\
\hline$E\left[\hat{N}_{m}\right]$ (average total measurements) & 2712.7 & 57 \\
\hline \hline
\end{tabular}

as

$$
\psi_{i}=E\left[u_{i}\right]-E\left[N_{m}\right] c_{m},
$$

then it can be clearly seen in Table $\mathrm{V}$ that it has negligible impact for our utilized experimentation probability but has significant impact for experimentation probability 1. As all of our work uses $\varepsilon \ll 1$, we can ignore the measurements cost in our results.

\section{CONCLUding REMARKS AND FUture DiRECTIONS}

We have used a game theoretic trial and error learning rule to design a distributed channel/bonding size selection method. The proposed method enables multiple users that compete for channel access with heterogeneous $\mathrm{CB}$ capabilities to select those channels/bonding sizes using which they can increase their likelihood of achieving higher data rates. We use Nash equilibrium (NE) as a solution concept and show that under the interference graph representation, distributed channel/bonding size selection can be modeled as a generalized ordinal potential game. This means that there exists at least one pure strategy NE. Our results show that the proposed method enables the users to be in a NE with a very high probability. Moreover, through our simulations we also show how well the achieved worst NE solution and the best NE solution performs as compared to a centralized solution. We also evaluate the performance of the proposed method in terms of several different performance metrics. We also present an implementation of the prototype of the proposed method on wireless open access research platform (WARP) nodes. Using experiments which involve real over-the-air wireless communications, it is shown that the proposed method allows the WARP nodes to perform channel/bonding size selections that maximize their payoff.

Our work presents an ITE-based learning solution for channel/bonding size selection problem under the scenarios where access points (APs) are distributedly deployed. In practice, AP deployments in unlicensed and other shared bands can be distributed, and/or coordinated, as hospitals, universities, or businesses choose to deploy a network of hundreds of APs within their premises. One possible extension for this work is to not only consider the distributed deployment scenarios but also study channel/bonding size selection solutions under various deployment scenarios. For example, a scenario where some APs are deployed by a coordinated entity while others are deployed distributedly. One limitation in existing studies is that the current channel/bonding size selection schemes do not study the impact where some distributed APs (which are not controlled by the centralized entity) are interfering and will not obey the allocations suggested by the centralized entity.

\section{REFERENCES}

[1] Cisco. (2015) 802.11ac: The fifth generation of WiFi. [Online]. Available: http://www.cisco.com/c/en/us/products/collateral/ wireless/aironet-3600-series/white_paper_c11-713103.html

[2] Y. Lu, H. He, J. Wang, and $\mathrm{S}$. Li, "Opportunistic spectrum access with channel bonding," in proceedings of Fourth International Conference on Communications and Networking, August 2009, pp. 1-5.

[3] Z. Khan, H. Ahmadi, E. Hossein, M. Coupechoux, L. A. DaSilva, and J. J. Lehtomaki, "Carrier aggregation/channel bonding in next generation cellular networks: Methods and challenges," IEEE Network Magazine, vol. 28, no. 6, pp. 34-40, November 2014.

[4] L. Deek, E. Garcia-Villegas, E. Belding, S.-J. Lee, and K. Almeroth, "Intelligent channel bonding in 802.11n WLANs," IEEE Transactions on Mobile Computing, vol. 13, no. 6, pp. 1242-1255, June 2014.

[5] Rice University WARP project. [Online]. Available: \{http://warp.rice. $\mathrm{edu} /\}$

[6] H. P. Young, "Learning by trial and error," Games and Economic Behavior, vol. 65, no. 2, pp. 626-643, March 2009.

[7] Rice University WARPLAB project for OFDM-based Wireless Communications System. [Online]. Available: \{http://warpproject.org/ trac/wiki/WARPLab/Examples/OFDM

[8] B. Bellalta, A. Checco, A. Zocca, and J. Barcelo, "On the interactions between multiple overlapping WLANs using channel bonding," IEEE Transactions on Vehicular Technology, vol. 65, no. 2, pp. 796-812, Feb 2016.

[9] Qualcomm. (2013) 1000x: More spectrum-especially for small cells. [Online]. Available: http://www.qualcomm.com/media/documents/ 1000x-more-spectrum-especially-small-cells

[10] A. L. Ramaboli, O. E. Falowo, and A. H. Chan, "Bandwidth aggregation in heterogeneous wireless networks: A survey of current approaches and issues," Journal of Network and Computer Applications, vol. 35, no. 6, pp. $1674-1690,2012$.

[11] F. Kaltenberger, F. Foukalas, O. Holland, S. Pietrzyk, S. Thao, and G. Vivier, "Spectrum overlay through aggregation of heterogeneous dispersed bands," in proceedings of European Conference on Networks and Communications (EuCNC), June 2014.

[12] P. Huang, X. Yang, and L. Xiao, "Dynamic channel bonding: Enabling flexible spectrum aggregation," IEEE Transactions on Mobile Computing, vol. 15, no. 12, pp. 3042-3056, Dec 2016.

[13] H. Salameh, M. Krunz, and D. Manzi, "Spectrum bonding and aggregation with guard-band awareness in cognitive radio networks," IEEE Transactions on Mobile Computing, vol. 13, no. 3, pp. 569-581, March 2014. 
[14] R. Karmakar, S. Chattopadhyay, and S. Chakraborty, "Dynamic link adaptation in IEEE 802.11ac: A distributed learning based approach," in IEEE 41st Conference on Local Computer Networks (LCN), Nov 2016, pp. 87-94.

[15] P. Hernandez-Leal, M. Kaisers, T. Baarslag, and E. Munoz de Cote, "A survey of learning in multiagent environments: Dealing with non-stationarity," 2017, arXiv:1707.09183. [Online]. Available: https://arxiv.org/abs/1707.09183

[16] R. Engelberg, A. Fabrikant, M. Schapira, and D. Wajc, "Best-response dynamics out of sync: Complexity and characterization," in Proceedings of the Fourteenth ACM Conference on Electronic Commerce. New York, NY, USA: ACM, 2013, pp. 379-396.

[17] N. Nisan, M. Schapira, and A. Zohar, "Asynchronous best-reply dynamics," in Internet and Network Economics. Springer Berlin Heidelberg, 2008, pp. 531-538.

[18] Z. Khan, J. Lehtomäki, S. Scott, Z. Han, M. Krunz, and A. Marshall, "Distributed and coordinated spectrum access methods for heterogeneous channel bonding," IEEE Transactions on Cognitive Communications and Networking, vol. 3, no. 3, pp. 267-281, Sept 2017.

[19] X. Chen and J. Huang, "Distributed spectrum access with spatial reuse," IEEE Journal on Selected Areas in Communications, vol. 31, no. 3, pp. 593-603, March 2013.

[20] T. Rappaport, Wireless Communications: Principles and Practice, 2nd ed. Prentice Hall PTR, 2001.

[21] J. Bendor, D. Mookherjee, and D. Ray, "Reinforcement learning in repeated interaction games," The B.E. Journal of Theoretical Economics, vol. 1 , no. 1 , pp. $1-44,2001$

[22] B. Guney, M. Richter, and M. Tsur, "Aspiration-based choice," Journal of Economic Theory, vol. 176, pp. 935 - 956, 2018.

[23] P. Reverdy, V. Srivastava, and N. E. Leonard, "Satisficing in multi-armed bandit problems," IEEE Transactions on Automatic Control, vol. 62, no. 8, pp. 3788-3803, Aug 2017.

[24] Z. Khan, J. Lehtomäki, E. Hossain, M. Latva-aho, and A. Marshall, "An FPGA-based implementation of a multifunction environment sensing device for shared access with rotating radars," IEEE Transactions on Instrumentation and Measurements, pp. 1-14, 2018, to appear. [Online]. Available: \{http://www.ee.oulu.fi/ zaheer/main_manuscript.pdf $\}$

[25] J. R. Marden, H. P. Young, G. Arslan, and J. S. Shamma, "Payoff based dynamics for multi-player weakly acyclic games," in Detailed version (online), March 2007-2008, pp. 1-35. [Online]. Available: http: //www.econ2.jhu.edu/people/young/PayoffBasedDynamicsMar08.pdf

[26] - "Payoff based dynamics for multi-player weakly acyclic games," in 46th IEEE Conference on Decision and Control, Dec 2007, pp. 3422 3427.

[27] H. Tembine, Distributed Strategic Learning for Wireless Engineers. CRC Press, 2012.

[28] D. Monderer and L. S. Shapley, "Potential games," Games and Economic Behavior, vol. 14, no. 1, pp. 124 - 143, 1996. [Online]. Available: http://www.sciencedirect.com/science/article/pii/S0899825696900445

[29] System Generator for DSP. [Online]. Available: \{https://www.xilinx. com/products/design-tools/vivado/integration/sysgen.html \}

[30] Xilinx. Polyphase Filter Bank Channelizer. [Online]. Available: \{https://www.xilinx.com/support/documentation/application_notes/ xapp1161.pdf

[31] C. Hunter, P. Murphy, and A. Sabharwal, "Real-time testbed implementation of a distributed cooperative MAC and PHY," in 44th Annual Conference on Information Sciences and Systems (CISS), March 2010, pp. 1-6.

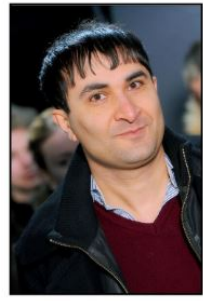

Zaheer Khan received his Dr.Sc in electrical engineering from the University of Oulu, Finland, and his M.Sc degree in electrical engineering from University College Borås, Sweden, in 2011 and 2007, respectively. Currently, he is an Adjunct Professor at the University of Oulu, Finland. He has also worked for a Tenure Track Lecturer position at the University of Liverpool, United Kingdom (20162017), and as a research fellow/principal investigator at the University of Oulu between 2011-2016. He was the recipient of the Marie Curie fellowship for 2007-2008. His research interests include implementation of advanced signal processing and wireless communications algorithms on Xilinx FPGAs and Zynq System-on-Chip (SoC) boards, application of game theory to model distributed wireless networks, prototyping access protocols for wireless networks, IoT location tracking systems, cognitive and cooperative communications, and wireless signal design.

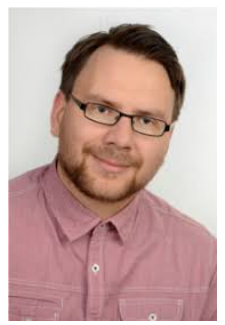

Janne Lehtomäki got his doctorate from the University of Oulu, Finland, in 2005. Currently, he is adjunct professor at the University of Oulu, Centre for Wireless Communications. He spent the fall 2013 semester at the Georgia Tech, Atlanta, USA, as a visiting scholar. Currently, he is focusing on spectrum measurements and terahertz band wireless communication. Dr. Lehtomäki has served as a guest associate editor for the IEICE TRANSACTIONS ON Communications Special Section (Feb. 2014 and July 2017) and as a managing guest editor for NANO Communication Networks Special Issue (June 2016). He was general co-chair of IEEE WCNC 2017 International Workshop on Smart Spectrum, TPC co-chair for IEEE WCNC 2015 and 2016 International Workshop on Smart Spectrum, publicity/publications co-chair for ACM NANOCOM 2015, 2016, 2017. He co-authored the paper receiving the Best Paper Award in IEEE WCNC 2012. He is editorial board member of PHYSICAL COMMUNICATION. 